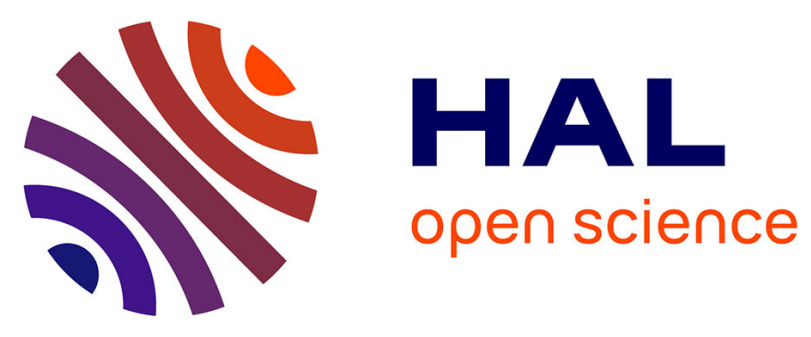

\title{
Deformation of Ni-Mn-Ga 7M modulated martensite through detwinning/twinning and forward/reverse intermartensitic transformation studied by in-situ neutron diffraction and interrupted in-situ EBSD
}

Naifu Zou, Zongbin Li, Y. D. Zhang, Weimin Gan, Bo Yang, Xiang Zhao, Claude Esling, Michael Hofmann, Liang Zuo

\section{To cite this version:}

Naifu Zou, Zongbin Li, Y. D. Zhang, Weimin Gan, Bo Yang, et al.. Deformation of Ni-Mn-Ga 7M modulated martensite through detwinning/twinning and forward/reverse intermartensitic transformation studied by in-situ neutron diffraction and interrupted in-situ EBSD. Acta Materialia, 2019, 174, pp.319-331. 10.1016/j.actamat.2019.05.054 . hal-03080183

\section{HAL Id: hal-03080183 \\ https://hal.univ-lorraine.fr/hal-03080183}

Submitted on 25 Oct 2021

HAL is a multi-disciplinary open access archive for the deposit and dissemination of scientific research documents, whether they are published or not. The documents may come from teaching and research institutions in France or abroad, or from public or private research centers.
L'archive ouverte pluridisciplinaire HAL, est destinée au dépôt et à la diffusion de documents scientifiques de niveau recherche, publiés ou non, émanant des établissements d'enseignement et de recherche français ou étrangers, des laboratoires publics ou privés.

\section{(ㅇ)(1) $\$$}

Distributed under a Creative Commons Attribution - NonCommerciall 4.0 International 


\section{Deformation of Ni-Mn-Ga 7M modulated martensite through detwinning/twinning and forward/reverse intermartensitic transformation studied by in-situ neutron diffraction and interrupted in-situ EBSD}

Naifu Zou ${ }^{\mathrm{a}, \mathrm{b}}$, Zongbin $\mathrm{Li}^{\mathrm{a}^{*}}$, Yudong Zhang ${ }^{\mathrm{b}, \mathrm{c}^{*}}$, Weimin Gan ${ }^{\mathrm{d}}$, Bo Yang ${ }^{\mathrm{a}}$, Xiang Zhao ${ }^{\mathrm{a}}$, Claude Esling ${ }^{\mathrm{b}, \mathrm{c}}$, Michael Hofmann $^{\mathrm{e}}$, Liang Zuo ${ }^{\mathrm{a}^{*}}$

${ }^{a}$ Key Laboratory for Anisotropy and Texture of Materials (Ministry of Education), Northeastern University, Shenyang 110819, China

b Laboratoire d'Étude des Microstructures et de Mécanique des Matériaux (LEM3), CNRS UMR 7239, Université de Lorraine, 57073 Metz, France

${ }^{c}$ Laboratory of Excellence on Design of Alloy Metals for low-mAss Structures (DAMAS), Université de Lorraine, 57073 Metz, France

d German Engineering Materials Science Centre (GEMS) at MLZ, Helmholtz-Zentrum Geesthacht (HZG), D-85748, Garching, Germany

${ }^{\text {e }}$ Forschungsneutronenquelle Heinz Maier-Leibnitz(FRMII), TU München, D-85747, Garching, Germany

*Corresponding author:

E-mail: yudong.zhang@univ-lorraine.fr. (Y. D. Zhang); lzuo@mail.neu.edu.cn (L. Zuo); lizb@atm.neu.edu.cn (Z. B. Li). 


\section{Abstract}

Shape memory alloys, especially the newly developed Ni-Mn-based heusler-type intermetallic compounds, exhibit specific mechanical responses to mechanical loading. Although the deformation behaviors have been studied for reducing the number of martensite variants, the mechanisms are not fully revealed. Thus in this work the compression process of twin-related 7M modulated martensite of Ni-Mn-Ga intermetallic compound was studied by in-situ neutron diffraction at macroscopic scale and by interrupted in-situ EBSD at microscopic scale. It is revealed that the mechanical response of the $7 \mathrm{M}$ martensite is featured by three states: a linear elastic-plastic state, a steady plastic state, and a second linear plastic state. The plastic deformation is initiated by the detwinning of the existing variants in the first linear state. It proceeds to the steady state by intensive detwinning of the these variants and by twinning of the remaining variants that result in the disappearance of the existing variants and the appearance of new variants, then by intermartensitic transformation to form non-modulated martensite (NM). These three shear processes are highly coordinated and compatible with the annihilation of the local incompatible strains by reverse intermartensitic transformation, which allows a steady progress of deformation and a continuous reorientation of the variants. The reorientation produces new twins with unfavorable orientations and limited deformation capacity, leading to a stress increase for further deformation. The present work provides comprehensive information on deformation mechanisms of Ni-Mn-Ga 7M martensite at each characteristic deformation step that is useful for mechanical simulation of deformation behaviors of intermetallic compounds. 
Keywords: Shape memory alloy; twinning/detwinning; Strain accommodation; Deformation behavior; Neutron diffraction

\section{Introduction}

Heusler-typed intermetallic compounds always show martensitic transformation and demonstrate various functionalities related either to the specific configuration of the resultant martensite or to the transformation process, such as shape memory effect $[1,2]$ and caloric effect (magneto- or elastocaloric) [3]. As these materials exhibit martensitic transformation that transforms the high temperature austenite to the low temperature martensite, various twin related crystallographic orientation variants of martensite can be produced from one parent orientation due to the symmetry of the parent austenite and the orientation relationship between the two phases.

For Ni-Mn-Ga Heusler-typed intermetallic compounds (the background information can be found in the next section), the superior functionalities are only attainable in single crystal state (especially in martensite state), thus the reduction of the number of the martensite variants is always imperative [4, 5]. Cyclic mechanical loading/unloading (the so-called mechanical training) has been commonly performed for such a purpose. As the atomic bonding of these materials are mainly of covalent type or ionic type, no dislocation slip is allowed for the plastic deformation. Thus their plastic deformation is mainly realized by twinning/detwinning and martensitic transformation, thus demonstrates specific deformation characteristics.

So far, many experimental investigations and theoretical simulations [6-23] have been 
conducted on mechanical training of Ni-Mn-Ga alloys with 5M, 7M and NM martensite. The training results were studied as further guidance for the training process. The investigations have mainly focused on the alloys with $5 \mathrm{M}$ or NM martensite due to the very low twinning stress of the former [6] and the high output strain of the latter [1]. Detailed twinning/detwinning processes have been well revealed for 5M [14] and NM martensite [15, $16,24]$ that have "hierarchically twinned" structures $[24,25]$. However for the $7 \mathrm{M}$ martensite without "hierarchically twinned" variant organization, the deformation behaviors have less been addressed.

Recently we investigated the deformed microstructure of Ni-Mn-Ga 7M martensite at the beginning state of a plastic deformation [26]. We found that due to the martensitic transformation twin related variants (We denoted such twins produced by phase transformation TrF-twins) are formed in the microstructure at room temperature. Intrinsically, each 7M martensite variant possesses various twinning systems for twinning and specific shear systems for intermartensitic transformation. Interestingly, the intermartensitic transformation was found to make important contribution to the macroscopic deformation. In addition, this process was in good coordination with the twinning process of the neighboring variants. Such stress-induced intermartensitic transformation has often been observed in $7 \mathrm{M}$ martensite [27-30]. However, the disappearance of the produced NM martensite has also been found after a complete deformation [18, 31], indicating the occurrence of reverse intermartensitic transformation during the late stage of deformation. However the mechanism of such change has not been revealed. Moreover, the stress-strain behavior of the Ni-Mn-Ga intermetallic compounds is always characterized by a superelasticity (a large strain achieved 
in the first linear stress-strain state) followed by a steady flow of strain without any recrystallization and then continued by a second linear stress increase. Such macroscopic behavior has not well been correlated with the microscopic deformation processes (detwinning/twinning or forward/reverse intermartensitic transformation).

In view of such a situation, we conducted the present work on deformation of Ni-Mn-Ga $7 \mathrm{M}$ martensite during a unidirectional compression process with an aim to fully uncover the deformation mechanisms of the Ni-Mn-Ga $7 \mathrm{M}$ martensite and to correlate the microscopic shear processes with the macroscopic mechanical behavior of the material. Special efforts have been made on characterizing the deformation process by in-situ neutron diffraction at macroscopic scale and by interrupted in-situ SEM-EBSD at microscopic scale. The obtained results in this work, on the one hand, provide comprehensive information to deepen the understanding of the mechanical behaviors of the intermetallic compounds that can serve as practical guidance for the mechanical training of these materials, and, on the other hand, also provide useful experimental data for simulation work on intermetallic compounds utilizing various mechanical models.

\section{Background information for Ni-Mn-Ga}

As one kind of typical Heusler-typed intermetallic compounds, the Ni-Mn-Ga compounds undergo martensitic transformation from a cubic austenite to various types of martensite (differentiated by their crystal structures), such as five-layer modulated monoclinic $(5 \mathrm{M})$, seven-layer modulated monoclinic (7M) and non-modulated tetragonal (NM) martensite depending on the composition of the alloy. Among them, 7M martensite is reported to possess a monoclinic incommensurate crystal structure and can be represented by a super 
cell containing ten sub cells along the $c$ axis [32]. The austenite transforms to the $7 \mathrm{M}$ martensite under the Pitsch orientation relationship (OR) [33]. Due to the symmetry of the cubic austenite and the OR, one orientation austenite can generate 24 crystallographic orientation variants of $7 \mathrm{M}$ martensite. The produced $7 \mathrm{M}$ martensite variants are in plate shape and grouped into 6 variant colonies [33]. Each colony contains 4 alternately distributed orientation variants designated as Variants A, B, C and D. The 4 variants are interrelated by three kinds of twin relations: Type-I twin (A and C or B and D), Type-II twin (A and B or C and D) and compound twin (A and D or B and C) [34]. The 7M martensite is metastable and can further transform to the NM martensite with temperature decrease or under an external load. Such a transformation from one kind of martensite to another is termed as intermartensitic transformation [35] and the reverse transformation is denoted reverse intermartensitic transformation.

\section{Experimental}

In the present work, polycrystalline Ni-Mn-Ga with nominal composition of $\mathrm{Ni}_{50} \mathrm{Mn}_{30} \mathrm{Ga}_{20}$ was prepared by arc-melting using high purity raw materials in a shielding gas of argon. The ingots were re-melted for four times to achieve composition homogeneity. Then the alloy was further directionally cast by means of Bridgeman method at a withdrawal rate of $50 \mu \mathrm{m} / \mathrm{s}$. The as-cast alloy was cut into rectangular parallelepiped samples with dimensions of $10 \times 5 \times 5 \mathrm{~mm}^{3}$ and with the longer edge parallel to the solidification direction (SD). The cut samples were further annealed at $1173 \mathrm{~K}$ for 24 hours under vacuum and air-cooled to the room temperature to homogenize the composition.

Unidirectional compression was performed under in-situ neutron diffraction 
measurement at room temperature, using the materials science diffractometer STRESS-SPEC at the Heinz Maier-Leibnitz Zentrum (MLZ) (Garching, Germany) [36]. The compression was conducted under the constant loading rate $(2 \mathrm{~N} / \mathrm{s})$. The macroscopic strain was determined from the crosshead displacement measured with a displacement sensor. Prior to the measurement, the sample was pre-compressed under a small stress of $3 \mathrm{MPa}$ to ensure a full contact between the crossheads and the sample. The neutron diffraction measurement was performed in-situ with the compression process. The incident beam was monochromatic with a wavelength of $2.1 \AA$ and a beam size of $5 \times 5 \mathrm{~mm}^{2}$. The neutron diffraction images were continuously recorded each $60 \mathrm{~s}$ using a two-dimensional (2D) detector with an opening window of $14^{\circ}(2 \theta)$. Fig. 1(a) illustrates the geometrical configuration of the measurement layout. Geometrical centers of the sample and the 2D detector were at the same height of the incident beam, and the neutron beam was injected horizontally to the sample, as illustrated in the figure. Thus the incident beam and the diffracted beam detected by the detector constituted the equatorial plane (also the horizontal pnae) in the figure. The angle between the compressive load direction (LD) and this equatorial plane was $\chi$. The LD tilted within the plane perpendicular to the equatorial plane and bisecting the angle between the incident beam and the detected diffracted beam, as shown in Fig. 1(a). This plane was also perpendicular to the reflecting plane of the diffracted beam detected by the detector. The rotation angle around the LD was $\omega$. The two angles, $\chi$ and $\omega$, defined the orientation of the sample with respect to the incident beam and the detector.

Pole figures of the initial sample and the deformed samples $(2 \%, 4 \%$ and $6 \%$ in reduction) were measured ex-situ by neutron diffraction to visualize the global texture 
evolution. The gauge volume of the samples for the pole figure measurements was 5 (parallel to the SD) $\times 5 \times 5 \mathrm{~mm}^{3}$. The diffraction patterns were extracted with the SteCa software from the recorded 2D diffraction images [37].

To follow the evolution of the microstructure and the crystallographic orientations of the microstructural constituents in the sample during the unidirectional compression, interrupted in-situ EBSD measurements were performed in a field-emission-gun scanning electron microscope (SEM, Jeol JMF6500-F) with an electron backscatter diffraction (EBSD) detecting system. Prior to the unidirectional compression, the samples were mechanically polished and then electrolytically polished with a solution of $20 \%$ nitric acid in methanol at temperatures below $273 \mathrm{~K}$ and at a voltage of $18 \mathrm{~V}$ for 10 seconds. The unidirectional compression in 4 steps was performed manually under controlled crosshead displacement using a home-made compression system, as shown in Fig. 1(b). The compressive load was applied by screwing forward the movable crosshead. The crosshead displacement was measured with a digital caliper (precision: $0.01 \mathrm{~mm}$ ). The compression was interrupted at the accumulative reductions of $2 \%, 3 \%, 4.5 \%$ and $5.5 \%$, respectively. Between the steps, the microstructural and crystallographic measurements were conducted in the same sample areas. To obtain statistical information, five EBSD maps with sizes of $80 \times 56 \mu \mathrm{m}^{2}$ were acquired.

To determine the in-situ neutron diffraction measurement setup, the initial texture of the as-annealed sample was measured. The present material was composed of $7 \mathrm{M}$ modulated martensite at room temperature. The as-annealed texture is represented by the pole figures of $\left\{\begin{array}{lll}\overline{1} & 2 & 10\end{array}\right\}_{7 \mathrm{M}},\left\{\begin{array}{lll}1 & 0 & 10\end{array}\right\}_{7 \mathrm{M}}$ and $\left\{\begin{array}{lll}1 & 2 & 10\end{array}\right\}_{7 \mathrm{M}}$ in Fig. 2, where the BD stands for the incident beam direction and the SD is parallel to the $\mathrm{LD}$. The three selected planes correspond to the 
twinning planes of the $7 \mathrm{M}$ martensite variants and are also the interfaces between the variants, as detailed in $§ 3.1$. As it can be seen in the figures, the texture of the sample is composed of several individual orientations that are characterized by the $\left\{\begin{array}{lll}\overline{1} & 210\end{array}\right\}_{7 \mathrm{M}}$ planes parallel to the SD and the $\left\{\begin{array}{lll}1 & 0 & 10\end{array}\right\}_{7 \mathrm{M}}$ planes perpendicular to the SD, and the $\left\{\begin{array}{lll}1 & 2 & 10\end{array}\right\}_{7 \mathrm{M}}$ plane at about $46^{\circ}$ to the SD. The orientations are not sharp but with a large spread around the SD.

From such a characteristic texture, the measurement setup of the in-situ neutron diffraction measurements during the unidirectional compression to monitor the three twinning systems was conceived. The pole figures in Fig. 2 indicate that the $\left\{\begin{array}{lll}1 & 2 & 10\end{array}\right\}_{7 \mathrm{M}},\left\{\begin{array}{lll}1 & 0 & 10\end{array}\right\}_{7 \mathrm{M}}$ and $\left\{\begin{array}{lll}1 & 2 & 10\end{array}\right\}_{7 \mathrm{M}}$ planes are located at $\left.\chi=90^{\circ}\left(\left\{\begin{array}{lll}\overline{1} & 2 & 10\end{array}\right\}_{7 \mathrm{M}}\right), 0^{\circ}\left(\left\{\begin{array}{lll}1 & 0 & 10\end{array}\right)\right\}_{7 \mathrm{M}}\right)$ and $44^{\circ}\left(\begin{array}{ll}1 & 2\end{array}\right.$ $10\}_{7 \mathrm{M}}$ ), respectively, thus three samples (denoted S1, S2 and S3) were used. The geometrical positions of the sample and the detector were given in Table 1. It is worth mentioning that due to the orientation spread of the planes the sample position was adjusted with respect to the determined position to capture abundant diffraction signal.

\begin{tabular}{cccc}
\hline Sample & $\chi\left(^{\circ}\right)$ & $2 \theta\left(^{\circ}\right)$ & $\omega\left(^{\circ}\right)$ \\
\hline S1 & 90 & 62 (for $\left\{\begin{array}{llll}\overline{1} & 2 & 10\end{array}\right\}_{7 \mathrm{M}}$ reflection) & \\
S2 & 0 & 42 (for $\left\{\begin{array}{llll}1 & 0 & 10\end{array}\right\}_{7 \mathrm{M}}$ reflection) & Adjustable \\
S3 & 44 & 62 (for $\left\{\begin{array}{lllll}1 & 2 & 10\end{array}\right\}_{7 \mathrm{M}}$ reflection) & \\
\hline
\end{tabular}

Table 1 Sample orientation represented by $\chi, 2 \theta$ and $\omega$ in polar coordinate system.

\section{Results and discussion}

\subsection{Initial microstructure}

Fig. 3(a) displays a large scaled initial microstructure. In the figure, the blue arrow indicates the SD, the red lines mark the initial austenite grain boundaries and the black lines 
outline some colonies of the $7 \mathrm{M}$ martensite. It is seen that the original austenite grains are in columnar shape and the martensite is in plate shape. Within each colony, the martensite plates are stretched roughly in the same direction. Fig. 3(b) shows an EBSD micrograph of the initial microstructure. In the figure the martensite variants are colored according to their crystallographic orientations (specifically according to their Euler angles) and the original austenite grain boundaries are outlined with the red lines and the martensite colonies with the black lines. One example colony enclosed in the yellow rectangle in Fig. 3(b) is further magnified and displayed with the corresponding $\left\{\begin{array}{llll}\overline{1} & 2 & 10\end{array}\right\}_{7 \mathrm{M},}\left\{\begin{array}{lll}1 & 0 & 10\end{array}\right\}_{7 \mathrm{M}}$ and $\left\{\begin{array}{lll}1 & 2 & 10\end{array}\right\}_{7 \mathrm{M}}$ plane pole figures in Fig. 3(c). It is seen that the colony contains 4 orientation variants denoted A, B, C and D. Variant pairs A-C and B-D are Type-I twins and separated by their common $\begin{cases}\overline{1} & 2\end{cases}$ $\left.{ }_{10}\right\}_{7 \mathrm{M}}$ planes that are also their Type-I twinning plane [38]. Variant pairs A-D and B-C are the compound twins and separated with the common $\left\{\begin{array}{lll}1 & 0 & 10\end{array}\right\}_{7 \mathrm{M}}$ planes that are also their compound twinning plane [38]. Such characteristic features are repeated in all the martensite colonies. The $\left\{\begin{array}{llll}1 & 2 & 10\end{array}\right\}_{7 \mathrm{M}}$ of Variant $\mathrm{C}$ is also marked in Fig. 3(c). This plane is the twinning plane of another kind of twin (as will be specified later). The EBSD pole figures of the three planes in Fig. 3(c) well reproduce one part of the poles in the corresponding neutron diffraction pole figures in Fig. 2. Moreover, the micrograph in Fig. 3(c) demonstrates that these poles are from the adjacent variants in the microstructure.

\subsection{In-situ neutron diffraction examination}

Fig. 4 shows the stress-strain curve of the unidirectional compression and the corresponding in-situ neutron diffraction patterns of the three samples acquired during the compression processes. The stress-strain curve in Fig. 4(a) demonstrates specific deformation 
behavior of the $7 \mathrm{M}$ martensite. From the starting point marked in black, the stress first increased almost linearly with the reduction from $0 \%$ to $2.2 \%$ (the red line part), then stayed more or less unchanged from $2.2 \%$ to $5.1 \%$ (the plateau part of the curve, i.e., the dark yellow part from $2.2 \%$ to $4 \%$ and the green line part from $4 \%$ to $5.1 \%$ ), demonstrating a steady stress state. After that, the stress increased again approximately linearly to the reduction of $6.3 \%$ (the cyan line part from $5.1 \%$ to $6.3 \%$ ). After the strain of the sample reached the designed value $6.3 \%$ (as marked with the olive point), the unloading process began (the blue line part) until completion. It should be mentioned that such a specific deformation feature has been observed in many Ni-Mn based intermetallic compounds [39].

The neutron diffraction patterns of S1 in Fig. 4(b $\left.b_{1}\right)$ well reveal the orientation change of the martensite during the deformation. It is seen that initially only the $\left\{\begin{array}{lll}\overline{1} & 210\end{array}\right\}_{7 \mathrm{M}}$ diffraction peak $\left(2 \theta=\sim 62^{\circ}\right)$ could be observed within the $2 \theta$ range of $55-69^{\circ}$. As described above, this plane is the twinning plane of the Type-I TrF-twins and is also the interface between the twin related variants (A-D and B-C) (Fig. 3(c)). When the deformation reached the first part of the plateau (2.2\% to $4 \%$, the dark yellow line part in Fig. 4(a)), the $\left\{\begin{array}{lll}\overline{1} & 210\end{array}\right\}_{7 \mathrm{M}}$ peak weakened by $\sim 44.9 \%$ and a peak at $2 \theta=60.4^{\circ}$ started to appear. This peak corresponded to the $\left\{\begin{array}{lll}1 & 1 & 2\end{array}\right\}_{\mathrm{NM}}$ peak, indicating the occurrence of the $7 \mathrm{M}$ to $\mathrm{NM}$ intermartensitic transformation. Such a structural transformation was evidenced in our previous study [24]. When the deformation reached the end of the plateau part (4\% to $5.1 \%$, the green line part in Fig. $4(a)$ ), the $\begin{cases}\overline{1} & 2\end{cases}$ $10\}_{7 \mathrm{M}}$ peak largely weakened by $\sim 84.1 \%$ with respect to the initial intensity, but the $\{11$ $2\}_{\mathrm{NM}}$ peak strengthened by $\sim 102.4 \%$ and a new peak appeared at $2 \theta=59.6^{\circ}$. This new peak corresponded to the $\left\{\begin{array}{lll}0 & 0 & 20\end{array}\right\}_{7 \mathrm{M}}$ peak. This indicated the occurrence of variant reorientation of 
the 7M martensite through twinning (this will further be specified with the interrupted in-situ EBSD investigation). When the deformation continued to the second linear part (the cyan line

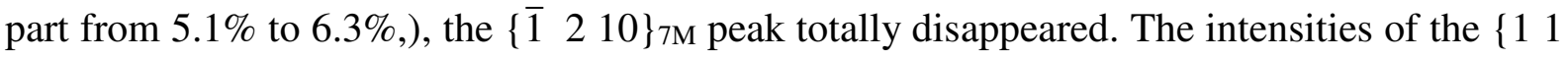
$2\}_{\mathrm{NM}}$ peak and the $\left\{\begin{array}{lll}0 & 020\end{array}\right\}_{7 \mathrm{M}}$ peak stayed almost stable till unloading (the olive point in Fig. 4(a)).

The similar situation appeared with the $\left\{\begin{array}{lll}1 & 0 & 10\end{array}\right\}_{7 \mathrm{M}}$ peak that corresponded to the compound TrF-twinning plane (one example shown in Fig. 3(c)). As shown by the neutron diffraction patterns of S2 in Fig. $4\left(b_{2}\right)$, the $\left\{\begin{array}{llll}1 & 0 & 10\end{array}\right\}_{7 \mathrm{M}}$ peak weakened $\sim$ by $21.9 \%$ in the first part of the plateau $(2.2 \%$ to $4 \%)$ in the stress-strain curve and disappeared in the second part (4\% to $5.1 \%$ ) (Fig. $\left.4\left(\mathrm{~b}_{2}\right)\right)$. Simultaneously, the $\left\{\begin{array}{lll}0 & 2 & 0\end{array}\right\}_{7 \mathrm{M}}$ and the $\left\{\begin{array}{lll}1 & 1 & 0\end{array}\right\}_{\mathrm{NM}}$ peaks appeared and strengthened, indicating the reorientation of the $7 \mathrm{M}$ variants through twinning/detwinning and the occurrence of the intermartensitic transformation during the deformation. In addition, the $\left\{\begin{array}{lll}1 & 0 & 10\end{array}\right\}_{7 \mathrm{M}}$ peak in the first part of the plateau $(2.2 \%$ to $4 \%)$ shifts slightly $\left(\sim 1^{\circ}\right)$ toward the higher $2 \theta$ with respect to that in the first linear part of the stress-strain curve, suggesting a decrease of the inter-planar spacing of the $\left\{\begin{array}{llll}1 & 0 & 10\end{array}\right\}_{7 \mathrm{M}}$ planes. As seen from Fig. 2(b) that in the initial state most of the $7 \mathrm{M}$ martensite variants were oriented with their $\left.\begin{array}{llll}1 & 0 & 10\end{array}\right\}_{7 \mathrm{M}}$ plane perpendicular to the compressive load, the decrease of the planar spacing of these planes suggested an elastic response of the martensite to the imposed compression.

Fig. 4(b $\left.b_{3}\right)$ displays the diffraction patterns of S3 acquired during the deformation process. In addition to the intensity change of the existing $7 \mathrm{M}$ peaks and the disappearance/appearance of the $7 \mathrm{M}$ peaks in Fig. $4\left(\mathrm{~b}_{1}\right)$ and $\left(\mathrm{b}_{2}\right)$, the intermartensitic transformation was revealed, as highlighted with the rectangular frame in Fig. $4\left(b_{3}\right)$ and further magnified in the insert in the 
figure. The $\left\{\begin{array}{lll}2 & 0 & 0\end{array}\right\}_{\mathrm{NM}}$ at $2 \theta=65.6^{\circ}$ initially appeared in the first part of the plateau $(2.2 \%$ to 4\%) of the stress-strain curve (Fig. 4(a)) (indicating the deformation induced intermartensitic transformation) but then greatly weakened by $\sim 76 \%$ in the second part (4\% to $5.1 \%$ ) and finally disappeared when the strain reached the second linear deformation regime $(5.1 \%$ to 6.3\%). The disappearance of this peak could be a result of reorientation or a reverse intermartensitic transformation. This will be clarified with the interrupted in-situ SEM-EBSD investigation.

\subsection{Interrupted in-situ EBSD examination}

Fig. 5 shows the EBSD micrographs acquired from one of the representative sample areas corresponding to the initial state $\left(\mathrm{a}_{1}\right)$, and the states after $2 \%\left(\mathrm{~b}_{1}\right), 3 \%\left(\mathrm{c}_{1}\right), 4.5 \%\left(\mathrm{~d}_{1}\right)$ and $5.5 \%\left(\mathrm{e}_{1}\right)$ reductions. The corresponding area fractions of the martensite variants with the standard deviations are also displayed in the figure. These deviations rather represent the area fraction spreads than errors. The four reductions corresponded to the first linear part (2\%), the beginning part (3\%) and the last part (4.5\%) of the plateau, and the second linear part $(5.5 \%)$ of the stress-strain curve in Fig. 4(a). The measured area corresponds to one martensite variant colony. One can see that before the deformation there were four plate-shaped $7 \mathrm{M}$ martensite variants, referred to as Variant A, B, C and D (Fig. 5(a $\left.\mathrm{a}_{1}\right)$ ). Orientation analysis demonstrated that the four variants were interrelated by three types of twin relations, i.e., Type-I twin (A-C and B-D), Type-II twin (A-B and C-D) and compound twin relationships (A-D and B-C), as frequently reported in the literature for the 7M Ni-Mn-Ga intermetallic compounds [40]. As these twins were produced by the austenite to $7 \mathrm{M}$ martensite transformation and existed before the compression, we denoted these $7 \mathrm{M}$ martensite variants 
TrF-twins to differentiate them from the twins produced by the deformation. The corresponding twinning elements of the TrF-twins are listed in Table A.1 in Appendix. The interfaces between the TrF-twins or variants were their $\mathrm{K}_{1}$ planes $\left(\left\{\begin{array}{lll}\overline{1} & 2 & 10\end{array}\right\}_{7 \mathrm{M}}\right.$ for the Type-I TrF-twins, $\left\{\begin{array}{lll}\overline{1.0621} & 29.3785\end{array}\right\}_{7 \mathrm{M}}$ for the Type-II TrF-twins and $\left\{\begin{array}{lll}1 & 0 & 10\end{array}\right\}_{7 \mathrm{M}}$ for the compound TrF-twins), as marked in Fig. 5(a $\left.\mathrm{a}_{1}\right)$. From Table A.1, one can find that the Type-I and Type-II TrF-twins possess the same magnitude of twinning shear. The twinning shears of the compound TrF-twins are the same but with much smaller magnitude, one order smaller than those of the Type-I and Type-II TrF-twins. Thus the contribution of the compound TrF-twins to the macroscopic deformation is negligible. If we only consider the strain contribution of the variants through twinning or detwinning to the external deformation, the variants with compound TrF-twin relation can be treated as one variant. From Fig. 5(a $\left.\mathrm{a}_{2}\right)$ one can see that the area fractions of Variant $\mathrm{A}+\mathrm{D}$ and Variant $\mathrm{B}+\mathrm{C}$ were similar, each pair being about $50 \%$. To estimate the activation possibilities of the detwinning of the existing variants (the TrF-twins), their Schmid Factors (SFs) were calculated with respect to the external compressive load and are listed in Table 2. Although the activation of the individual shear systems could be strongly related to their local stress state, we could assess that the applied external force imposed the major influence at the beginning of the deformation. The results showed that the detwinning systems of all the three kinds of TrF-twins had small SFs, but the detwinning of the compound TrF-twin had relatively higher SFs and should be easy to be activated.

\begin{tabular}{ccc}
\hline & & SF \\
\hline & Type-I & $-0.0105 \sim 0.0094$ \\
Detwinning of TrF-twin & Type-II & $-0.0045 \sim 0.0029$ \\
& compound & $-0.1346 \sim 0.1192$ \\
& 14 &
\end{tabular}


Twinning of DeF-twin $\quad$ Type-I $\quad 0.4657 \sim 0.4887$

Shear of intermartensitic transformation

Table 2 SFs of the detwinning systems of TrF-twins, twinning system of DeF-twins and the shear system of the intermartensitic transformation.

Fig. 5(b $\left.b_{1}\right)$ shows the microstructure in the same sample area after the $2 \%$ reduction, corresponding to the end of the first linear part of the stress-strain curve (Fig. 4(a)). Compared with those of the initial state in Fig. 5(a $\left.a_{2}\right)$, the area fractions of the variants changed with no new variant detected, as seen in Fig. 5(b $\left.b_{2}\right)$. The fraction of Variant A increaseed (Fig. 5(b $\left.b_{2}\right)$ ). The increase was realized by both the lengthening of $\mathrm{A}$ at the expense of $\mathrm{D}$ (as indicated by the blue arrow in Fig. 5(b $\left.b_{1}\right)$ ) and by the thickening at the expenses of $C$ (as indicated by the red arrow in Fig. 5(b $\left.b_{1}\right)$ ) and B (as indicated by the brown arrow in Fig. 5(b $\left.b_{1}\right)$ ). As described above, A and D were compound TrF-twin related, A and C Type-I TrF twin related, and A and B Type-II TrF-twin related, thus the growth of Variant A was done through the reverse process of the three twinning processse, i.e., the detwinning processes. Similar processes also happened to Variant B. Although the fraction of Variant B did not change much (Fig. 5( $\left.b_{2}\right)$ ), from Fig. 5(b $\left.b_{1}\right)$ one could see that some of the variants slightly thickened. This thickening was at the expense of Variant D (Variant B and D were Type-II TrF-twin related). However, the detwinning did not happen homogeneously for all Variant A and Variant B. In some locations, it occurred more intensively than in the other locations, as reflected by the enlarged standard deviations of the area fractions of these variants. The area fraction change of the variants 
revealed the occurrence of detwinning processes of the TrF-twins through twin boundary motion, evidencing the activation of the detwinning of the TrF-twins, especially the detwinning of the compound TrF-twins. This was consistent with the SFs of the TrF-twins. Thus in the first linear part of the stress-strain curve, the alloy responded to the external load by a mixed elastic-plastic deformation. The plastic part was mainly realized by the detwinning of the existing TrF-twins. This well uncovered the pseudo-elasticity of this kind of intermetallic compounds and the nature of the superelasticity.

As the sample was further deformed to the reduction of $3 \%$, i.e., the first half of the plateau part of the stress-strain curve (Fig. 4(a)), the microstructure changed drastically. Detwinning of the TrF-twins happened intensively. Variant D totally disappeared and only limited area fraction of Variant B remained. However, the area fractions of Variant A and C increased. Variant A and C were Type-I TrF-twin related. This confirmed that the twinning resistance of the Type-I TrF-twins was much higher than those of the Type-II and the compound TrF-twins, as reported in Ref. [41]. Accompanying the detwinning process between the existing variants with TrF-twin relations, the $\left\{\begin{array}{llll}1 & 2 & 10\end{array}\right\}_{7 \mathrm{M}} /<\overline{11.045} 10$

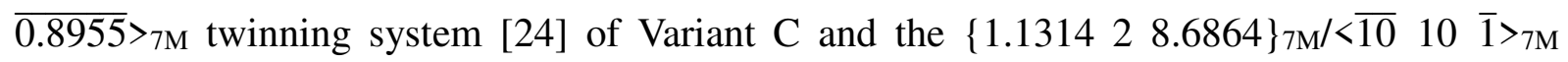

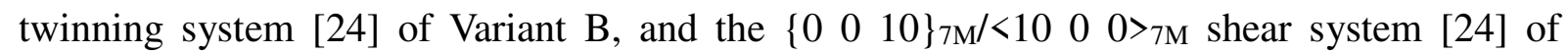
Variant A were activated. Due to the twinning process of Variant C and Variant B (as indicated with the black arrows in Fig. 5( $\left.\mathrm{c}_{1}\right)$ ), a new $7 \mathrm{M}$ martensite variant, named Variant $\mathrm{E}$ (in pink in Fig. 5( $\left.\mathrm{c}_{1}\right)$ ), was produced. Variant E was related with Variant $\mathrm{C}$ by a Type-I twin relation and with Variant B by a Type-II twin relation. We denoted these new twins DeF-twins to distinguish them from the TrF-twins. The corresponding twinning elements of these new 
twins (the DeF-twins) were determined and are displayed in Table A.2 in Appendix. Comparing the twinning elements of the DeF-twins with those of the TrF-twins (Table A.2 with Table A.1), one could find that the twinning shear of the DeF-twins was about one half of that of the TrF-twins, indicating that the deformation capacity of these twins was smaller than the TrF-twins. The activation of the $\left\{\begin{array}{lllll}0 & 0 & 10\end{array}\right\}_{7 \mathrm{M}} /<1 \begin{array}{llll}0 & 0 & 0\end{array}>_{7 \mathrm{M}}$ shear system of Variant A did not form new 7M variant, instead, it induced the intermartensitic transformation of Variant $\mathrm{A}$ and transformed Variant A into NM (in orange in Fig. 5( $\left.c_{1}\right)$ and $\left(c_{2}\right)$ ). The SF of the DeF-twinning systems and the intermartensitic transformation shear system under the compressive load were also calculated and displayed in Table 2. It is seen that although these systems possessed relatively larger SFs, none was activated when the detwinning of the TrF-twins (with lower SFs) happened intensively. This suggests that the twinning resistance or the Critical Resolved Shear Stress (CRSS) of these systems should be much higher. From the microstructure in Fig. 5( $\left.\mathrm{c}_{1}\right)$, we could see that the NM martensite and Variant E were formed in continuity one next to the other and repeatedly, changing the initial plate-like microstructure to a grid-like one. The orientation relationship (OR) between Variant A and the NM is $\left.\begin{array}{llll}0 & 0 & 10\end{array}\right\}_{7 \mathrm{M}} / /\left[\begin{array}{lll}1 & \overline{1} & 2\end{array}\right\}_{\mathrm{NM}}$ and $\left\langle\begin{array}{lll}10 & 0 & 0\end{array}>_{7 \mathrm{M}} / /<1 \quad \overline{1} \quad \overline{1}>_{\mathrm{NM}}\right.$. This orientation relationship is typical for the intermartensitic

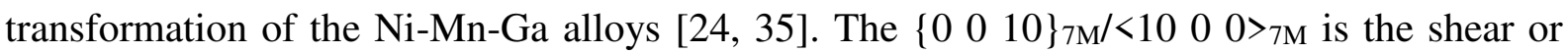
shuffling system for the intermartensitic transformation [35]. These results evidenced that the first part of the plastic deformation was realized by intensive detwinning of the existing TrF-twins (Type-I, Type-II and compound), the activation of the twinning of the Type-I and Type-II DeF-twins and the intermartensitic transformation.

When the compression proceeded to $4.5 \%$, i.e., the second half of the plateau part of the 
stress-strain curve (Fig. 4(a)), drastic boundary motion happened (Fig. 5(d $\left.\mathrm{d}_{1}\right)$ ) between Variant $\mathrm{C}$ and $\mathrm{E}$ through twinning and between Variant $\mathrm{A}$ and the NM martensite through intermartensitic transformation, resulting in the area fraction decrease of Variant $\mathrm{C}$ and $\mathrm{A}$ and the area fraction increase of Variant E and of the NM martensite, as displayed in Fig. $5\left(\mathrm{~d}_{2}\right)$. Moreover, the boundary between Variant $\mathrm{C}$ and $\mathrm{E}$ and that between Variant $\mathrm{A}$ and the NM martensite moved coordinately, keeping the twin and the phase boundaries in continuity. This consistent movement was the consequence of the consistent shear deformation through the DeF-twinning of Variant $\mathrm{C}$ and the shuffling of Variant A and the geometrical consistency of the two systems (the twinning system and the shuffling system). For an easy reference, the deformation gradient tensors representing the lattice deformation of the two shear systems, expressed in the macroscopic sample coordinate system (as used in Fig. 5 where the $\mathrm{X}$ axis is the compression axis) is given in Table 3 . In the two tensors, the values of $\varepsilon_{11}$ are smaller than 1, that of $\varepsilon_{22}$ larger than 1 and that of $\varepsilon_{33}$ close to 1 . This indicated that the Type-I DeF-twinning of Variant $\mathrm{C}$ and the shuffling of Variant A produced a contraction in the LD, and an elongation and a slight contraction in the other perpendicular directions. These characteristics revealed that the twinning of Type-I DeF-twin and the shuffling well accommodated the macroscopic strain and also very compatible between them two. The similar shear elements $\varepsilon_{\mathrm{ij}}$ in the two tensors also demonstrate that the shear was consistent in forming the new $7 \mathrm{M}$ variants and in forming the NM martensite. Such geometrical consistency of the two systems (DeF-twinning and shuffling) allowed the two deformation processes progressing in continuity until the two old variants ( $\mathrm{C}$ and $\mathrm{A})$ were fully replaced. This accounted for the steady flow character of the deformation of the present material. 
Interestingly, such a deformation character was normally observed in the alloys with metallic bonds during their isothermal deformation process in which two processes, i.e., work hardening by dislocation multiplication and work softening by recovery and recrystallization, were in balance. However, for the present material the mechanism for the steady flow was very different. It was by a continuous process of highly coordinated twinning and intermartensitic transformation.

\begin{tabular}{cccl}
\hline Variant & \multicolumn{3}{c}{ Deformation gradient tensor, $\varepsilon_{i j}$} \\
\hline \multirow{2}{*}{ C (to E) } & 0.9521 & $\overline{0.0263}$ & 0.0072 \\
& 0.0881 & 1.0484 & $\overline{0.0132}$ \\
& 0.0033 & 0.0018 & 0.9995 \\
A (to NM) & 0.9460 & $\overline{0.0087}$ & 0.0158 \\
& $\underline{0.0859}$ & $\overline{1.0572}$ & $\overline{0.0063}$ \\
\hline
\end{tabular}

Table 3 Deformation gradient tensors of Type-I DeF-twinning of Variant $\mathrm{C}$ and shuffling of Variant $\mathrm{A}$ in the macroscopic sample coordinate frame. The compressive load was applied along the $\mathrm{X}$ axis.

Another spectacular phenomenon was the noticeable appearance of Variant $\mathrm{O}$ (in jacinth in Fig. 5(c) to (e)) formed in the NM martensite regions at the first half of the plateau part of the stress-strain curve (Fig. 4(a)). It shared a straight interface with Variant E, as seen in Fig. 5( $\left.\mathrm{d}_{1}\right)$. Moreover, it grew, on the one hand, together with the NM martensite into Variant A and, on the other hand, into the host NM martensite, with the deformation (Fig. 5( $\left.c_{1}\right)$ and $\left(d_{1}\right)$ ). Such characteristics suggest that the formation of Variant $\mathrm{O}$ was realized by the reverse intermartensitic transformation from the $\mathrm{NM}$ martensite back to the $7 \mathrm{M}$ martensite. By orientation calculations, we found that the two kinds of martensite possessed the OR of intermartensitic transformation. It was $\left(\begin{array}{lll}0 & 0 & 10\end{array}\right)_{7 \mathrm{M}} / /\left(\begin{array}{lll}1 & 1 & 2\end{array}\right)_{\mathrm{NM}}$ and $\left.\left[\begin{array}{llll}10 & 0 & 0\end{array}\right]_{7 \mathrm{M} / /[} \begin{array}{lll}1 & 1 & 1\end{array}\right]_{\mathrm{NM}}$ with a 
deviation angle of the OR planes and OR directions of $0.32^{\circ}$ and $0.30^{\circ}$, respectively. This further confirmed that the reverse martensitic transformation was possible by the reverse shuffling on another system in the same family. By comparing Fig. 5( $\left.c_{1}\right)$ and $\left(d_{1}\right)$, we also noticed that Variant $\mathrm{E}$ and $\mathrm{C}$ slightly thickened at the expense of the respective $\mathrm{NM}$ and Variant A, suggesting that the reverse intermartensitic transformation also happened from NM to Variant E. Crystallographic examination revealed that the intermartensitic transformation OR also existed between these two. It was $\left(\begin{array}{lll}0 & 0 & 10\end{array}\right)_{7 \mathrm{M}} / /\left(\begin{array}{lll}1 & 1 & 2\end{array}\right)_{\mathrm{NM}}$ and $\left[\begin{array}{lll}10 & 0 & 0\end{array}\right]_{7 \mathrm{M}} / /\left[\begin{array}{lll}1 & 1 & 1\end{array}\right]_{\mathrm{NM}}$ with deviation angles between the respective OR planes and the OR directions of $0.67^{\circ}$ and $0.70^{\circ}$. Thus the difference between these two reverse intermartensitic transformation processes (NM to Variant E with slight amount and NM to $\mathrm{O}$ with large amount) should be studied to figure out the reason why NM martensite preferred to transform to Variant $\mathrm{O}$ of the 7M martensite rather than to Variant E.

With the ORs, the reverse shuffling process (intermartensitic transformation) from NM martensite to Variant $\mathrm{O}$ and to Variant $\mathrm{E}$ can be visualized by the atomic correspondences between each pair, as displayed in Fig. 6. The lattice deformation in the reverse intermartensitic transformation to form $7 \mathrm{M}$ variants can be regarded as an average shear to change the tetragonal NM lattice to the average lattice of the monoclinic martensite plus a lattice modulation to eventually form the $7 \mathrm{M}$ modulated martensite. Thus the average shear distinguishes the lattice deformation to form the two $7 \mathrm{M}$ variants $(\mathrm{O}$ and $\mathrm{E})$, as they have the same lattice modulations. The calculated average shears to form Variant $\mathrm{O}$ and $\mathrm{E}$ were 0.1362 and 0.2505 , respectively. Obviously, the reverse shuffling to Variant $\mathrm{O}$ was much smaller and thus much easier. Therefore the NM martensite preferred to transform to Variant $\mathrm{O}$. 
To further study the contribution of the formation of Variant $\mathrm{O}$ to the macroscopic deformation, the reverse intermartensitic transformation was further correlated with the macroscopic deformation. The contribution of this process was evaluated by the corresponding deformation gradient tensor as given in Table 4 . In the tensor, the value of $\varepsilon_{11}$ is slightly higher than 1, meaning no contribution to the macroscopic deformation. Moreover, the deformation gradient tensor has $\varepsilon_{22}$ smaller than 1 and $\varepsilon_{33}$ larger than 1 which is opposite to the deformation gradient tensors of Type-I DeF-twinning of Variant $\mathrm{C}$ and of the shuffling of Variant A, as displayed in Table 3. This suggests that the reverse martensitic transformation from the NM to Variant $\mathrm{O}$ was not compatible with the macroscopic deformation. It should be induced by local constraints. By comparing the deformation gradient tensor of Variant $\mathrm{C}$ to $\mathrm{E}$ and that of Variant A to NM in Table 3, one can find that although the deformations of the Type-I DeF-twinning of Variant C and the shuffling of Variant A are largely consistent, small difference exists between the corresponding elements in the two tensors. These differences were accumulative with the growth of Variant E and the NM martensite and became un-ignorable when the two constituents exceeded certain sizes. Thus local accommodation should be required. The reverse intermartensitic transformation could be in this purpose. We further calculated the deformation gradient tensor from Variant A to $\mathrm{O}$ via the intermartensitic transformation and the reverse intermartensitic transformation. The results are given in Table 5. For an easy comparison, the deformation gradient tensor describing the transformation from Variant $\mathrm{C}$ to $\mathrm{E}$ by Type-I DeF-twinning is copied from Table 3. One can found that excellent matches happen to the three corresponding elements in the first row in the two tensors. This row contains one normal strain and two shear strains in the compression 
direction. The excellent matches indicate that the occurrence of the reverse intermartensitic transformation was indeed to accommodate the accumulated incompatible strains between the growths of Variant E and NM martensite. However, the matches between the corresponding elements in the second and the third rows in the two tensors were not as excellent as those of the first row, meaning that the accommodations in the directions perpendicular to the compression direction were not so good. This may explain why the replacement of the NM martensite by Variant $\mathrm{O}$ was not always complete, as shown in Fig. $5\left(\mathrm{~d}_{1}\right)$. Variant $\mathrm{O}$ fully stretched in the length direction of the NM martensite that was close to the compression direction (the $\mathrm{X}$ axis) but did not in the other perpendicular directions (the $\mathrm{Y}$ and $\mathrm{Z}$ axes).

\begin{tabular}{cccc}
\hline Variant & \multicolumn{4}{c}{ Deformation gradient tensor, $\varepsilon_{i j}$} \\
\hline \multirow{3}{*}{ NM to O } & $\overline{1.0084}$ & $\overline{0.0046}$ & $\overline{0.0039}$ \\
& 0.0342 & 0.9366 & $\overline{0.0523}$ \\
& 0.0290 & 0.0684 & 1.0666 \\
\hline
\end{tabular}

Table 4 Deformation gradient tensors of reverse intermartensitic transformation (NM to Variant $\mathrm{O}$ ) in the macroscopic sample coordinate frame. The compressive load was applied along the $\mathrm{X}$ axis.

\begin{tabular}{cccc}
\hline Variant & \multicolumn{3}{c}{ Deformation gradient tensor, $\varepsilon_{i j}$} \\
\hline \multirow{3}{*}{ A to NM then to O } & 0.9535 & $\overline{0.0135}$ & 0.0121 \\
& 0.0483 & 0.9915 & $\overline{0.0581}$ \\
& 0.0282 & 0.0502 & 1.0544 \\
C to E & 0.9521 & $\overline{0.0263}$ & 0.0072 \\
& 0.0881 & 1.0484 & $\overline{0.0132}$ \\
& 0.0033 & 0.0018 & 0.9995 \\
\hline
\end{tabular}

Table 5 Deformation gradient tensors of Variant A to NM and then to Variant O, and Variant C to Variant E in the macroscopic sample coordinate frame. The compressive load was applied along the $\mathrm{X}$ axis. 
In view of the appearance of Variant $\mathrm{O}\left(\mathrm{Fig} .5\left(\mathrm{c}_{2}\right)\right.$ and $\left.\left(\mathrm{d}_{2}\right)\right)$, coherent interfaces were expected between Variant $\mathrm{O}$ and the adjacent variants separated by straight interfaces. In addition to the NM martensite, Variant $\mathrm{O}$ was also in neighborhood with Variant $\mathrm{E}$ and $\mathrm{A}$. Thus the respective orientation relationships were calculated with the measured Euler angles by EBSD. The results are given in Table 6. It can be seen that between Variant $\mathrm{O}$ and Variant E (separated by straight interface) there exist two $180^{\circ}$ rotations, suggesting that the two variants are in compound twin relation. This compound twin is not the same as that of the compound TrF-twin. To fully identify the twinning features of this twin, the twinning elements were determined using the method in Ref. [42]. The results are displayed in Table 7. In addition to the differences in twinning plane and twinning direction and their conjugates, the noticeable difference happens to the twinning shear. The shear magnitude of this compound twin is 10 times higher than that of the compound TrF-twin (Table A.1) and is close to those of the Type-I and Type-II DeF-twins (Table A.2). Here we also denote this twin compound DeF-twin. Further analysis on the interface between Variant $\mathrm{O}$ and Variant $\mathrm{E}$ using the indirect two trace method [43] revealed that the O-E interface coincided largely with the twinning plane with a small deviation of about $0.47^{\circ}$, as illustrated in Fig. 7 . Thus this interface should be coherent. This explains why the O-E interfaces were always straight. However, the orientation relationship between Variant $\mathrm{O}$ and Variant A were not clearly defined. The two variants were related to each other with a relation between the Type-I and the Type-II DeF-twins. The ambiguous twin relation between these two variants further confirmed that the growth of Variant $\mathrm{O}$ into Variant A was realized by two steps, firstly intermartensitic transformation of Variant $\mathrm{A}$ to $\mathrm{NM}$ and then reverse intermartensitic 
transformation from NM to Variant $\mathrm{O}$.

Clearly the formation of Variant $\mathrm{O}$ had two folds of importance. The first was that it annihilated the local incompatible strain created by the formation of Variant $\mathrm{E}$ from Variant $\mathrm{C}$ through Type-I DeF-twinning and the formation of NM from Variant A through intermartensitic transformation. This further ensured the steady deformation process of the present material by a continuous process of highly coordinated twinning and intermartensitic transformation until the disappearance of the remaining variants ( $\mathrm{C}$ and $\mathrm{A})$. The second was that it created a new compound twin relation with Variant E. This twin has relatively higher twinning resistance and lower shear magnitude.

This part of the results evidenced that the last part of the steady plastic deformation was realized by intensive DeF-twinning and intermartensitic transformation. During these processes, local incompatible strain was accumulated and thus the reverse martensitic transformation was initiated. In general, the twinning process and the intermartensitic transformation proceeded in a strain compatible way without much local resistance from the incompatible strains and therefore the plastic deformation was still in a steady way.

\begin{tabular}{|c|c|c|}
\hline Variant pair & Misorientation angle $\left({ }^{\circ}\right)$ & $\begin{array}{l}\text { Plane normal or direction parallel to } d \text { (in } \\
\text { the lattice basis of the } 7 \mathrm{M} \text { modulated } \\
\text { martensite) }\end{array}$ \\
\hline \multirow{2}{*}{$\mathrm{O}-\mathrm{E}$} & 179.89 & {$\left[\begin{array}{lllll}10 & 0.006 & 0.003\end{array}\right] 0.201^{\circ}$ from $\left[\begin{array}{lll}10 & 0 & 0\end{array}\right]$} \\
\hline & 179.91 & $\left(\begin{array}{lll}\overline{0.003} & \overline{0.001} & 10\end{array}\right) 0.203^{\circ}$ from $\left(\begin{array}{lll}0 & 0 & 10\end{array}\right)$ \\
\hline \multirow[b]{2}{*}{$\mathrm{O}-\mathrm{A}$} & 89.83 & \\
\hline & 179.81 & 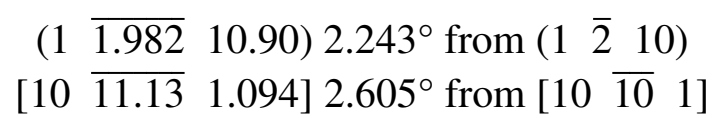 \\
\hline
\end{tabular}

Table 6 Misorientation (in rotation angle and axis) between Variant $\mathrm{O}$ and Variant $\mathrm{E}$, and 
Variant $\mathrm{O}$ and Variant $\mathrm{A}$.

\begin{tabular}{|c|c|}
\hline Variant pairs & O-E (compound DeF) \\
\hline$K_{1}$ & $\left(\begin{array}{lll}0 & 0 & 10\end{array}\right)$ \\
\hline$K_{2}$ & $\left(\begin{array}{lll}1 & 0 & 0\end{array}\right)$ \\
\hline$\eta_{1}$ & {$\left[\begin{array}{lll}10 & 0 & 0\end{array}\right]$} \\
\hline$\eta_{2}$ & {$\left[\begin{array}{lll}0 & 0 & 1\end{array}\right]$} \\
\hline$P$ & $\left(\begin{array}{lll}0 & \overline{1} & 0\end{array}\right)$ \\
\hline$s$ & 0.1143 \\
\hline
\end{tabular}

Table 7 Twinning elements $\left(K_{1}, K_{2}, \eta_{1}, \eta_{2}, P\right.$ and $\left.s\right)$ of the compound DeF-twin in Table 6.

When the deformation proceeded to $5.5 \%$ in reduction, i.e., the second linear part of the stress-strain curve (Fig. 4(a)), Variant A and Variant C almost disappeared through Type-I DeF-twinning (Variant $\mathrm{C}$ to Variant $\mathrm{E}$ ) and the intermartensitic transformation (Variant $\mathrm{A}$ to $\mathrm{NM}$ ) and the reverse intermartensitic transformation (NM to Variant $\mathrm{O}$ ), as shown in Fig. 5( $\left.\mathrm{e}_{1}\right)$ and $\left(\mathrm{e}_{2}\right)$. At this deformation state, the area fractions of Variant $\mathrm{E}$ and $\mathrm{O}$ increased but that of the NM decreased. This confirmed that the peak intensity decrease of the NM martensite in the neutron diffraction pattern in the second plateau part of the stress-strain curve in Fig. 4(a) was originated from the reverse intermartensitic transformation rather than NM variant reorientation. The area fraction increase of Variant E was realized not only by the Type-I DeF-twinning from Variant $\mathrm{C}$ to Variant $\mathrm{E}$ but also from compound DeF-twinning from Variant $\mathrm{O}$ to Variant $\mathrm{E}$ and the reverse intermartensitic transformation from NM to Variant $\mathrm{E}$. As these shear systems had lower SFs $(<0.02)$ and smaller shear magnitudes that represent higher twinning resistances, the further increase of the deformation strain required much higher stress level compared with that in the plateau part. Thus the stress-strain curve 
experienced a second linear part.

\subsection{Texture evolution}

The above deformation processes resulted in the orientation change of martensite variants. Fig. 8(a) and (b) show the EBSD $\left\{\begin{array}{lll}0 & 2 & 0\end{array}\right\}_{7 \mathrm{M}}$ pole figures of the respective initial state $(0 \%)$ and final deformed state $(-5.5 \%)$. It can be clearly seen that the $\left\{\begin{array}{lll}0 & 2 & 0\end{array}\right\}_{7 \mathrm{M}}$ planes of the four original variants are approximately parallel to the $\mathrm{X}$ axis (the compression direction, $\mathrm{LD}$ ). In detail, Variant A and Variant D were orientated with their $\left\{\begin{array}{lll}0 & 2 & 0\end{array}\right\}_{7 \mathrm{M}}$ planes almost perpendicular to the $\mathrm{Z}$ axis, whereas Variant $\mathrm{B}$ and Variant $\mathrm{C}$ to the $\mathrm{Y}$ axis. As the deformation progressed to the reduction of $5.5 \%$, the original variants (A, B, C and D) were all consumed by the new variants $(\mathrm{E}$ and $\mathrm{O})$. The $\left.\begin{array}{llll}0 & 2 & 0\end{array}\right\}_{7 \mathrm{M}}$ planes of the new variants appeared to be perpendicular to the LD, as shown in Fig. 8(b), forming a kind of $\langle 020\rangle_{7 \mathrm{M}}$ fiber texture with the compression direction (LD) as the fiber axis.

To further investigate if such texture change happened globally, i.e., if the same deformation processes visualized by the local EBSD examinations happened globally, through-volume neutron diffraction pole figure measurements were performed at the initial un-deformed state and the deformed states with reductions of $2 \%, 4 \%$ and $6 \%$, respectively. The corresponding $\left\{\begin{array}{lll}0 & 2 & 0\end{array}\right\}_{7 \mathrm{M}}$ pole figures are displayed in Fig. 9 . The four pole figures clearly show the similar orientation evolution from a texture with the $\left\{\begin{array}{lll}0 & 2 & 0\end{array}\right\}_{7 \mathrm{M}}$ poles located at the outer circle of the pole figure to a fiber texture with the $\left\{\begin{array}{lll}0 & 2 & 0\end{array}\right\}_{7 \mathrm{M}}$ poles located at the center of the pole figure. This meant that after the compression all the variants formed in the

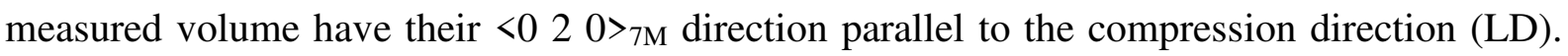
This result indicated that the deformation proceeded through detwinning, twinning, 
intermartensitic transformation and the reverse intermartensitic transformation revealed by the local in-situ EBSD investigation in the present work is representative for the whole material.

\section{Summary}

The microstructure evolution and the crystallographic orientation change during a unidirectional compression in a Ni-Mn-Ga alloy prepared by directional solidification were investigated by in-situ neutron diffraction measurements at macroscopic scale and by interrupted in-situ EBSD measurement at microscopic scale in the present work. It was revealed that each $7 \mathrm{M}$ modulated martensite variant of the Ni-Mn-Ga intermetallic compound possessed various shear systems allowing reversible processes of twinning (twinning and detwinning) and reversible intermartensitic transformation (forward and reverse). The plastic deformation of the Ni-Mn-Ga alloys in their martensitic state was realized by the activation of the various detwinning processes of the TrF-twins and the twinning of DeF-twins and intermartensitic transformation. The twinning and intermartensitic transformation processes were highly compatible to the macroscopic strain and to each other. The local incompatible strains accumulated at large deformation amount were further annihilated by reverse intermartensitic transformation. These processes allowed total reorientation of the initial variants and ensured a large steady stress-strain regime (plateau part). The orientations of the new variants set their shear systems in the unfavorable orientations with respect to the external load. Further deformation was realized by the shear processes (twinning and shuffling) with higher resistances and lower strain capacities, giving rise to the second line part of the stress-strain curve. The results of the present work provided detailed deformation mechanism information of $7 \mathrm{M} \mathrm{Ni-Mn-Ga} \mathrm{martensite} \mathrm{and} \mathrm{their} \mathrm{mechanical} \mathrm{responses} \mathrm{to} \mathrm{the}$ 
external loading. These results also provided basic experimental data in view of mechanical simulation work for intermetallic compounds, especially for shape memory alloys.

\section{Acknowledgements}

This work was supported by the European Commission under the 7th Framework Program through the 'Research Infrastructures' action of the 'Capacities' Program, NMI3-II Grant No. 283883. Financial supports from the National Natural Science Foundation of China (Grant Nos. 51431005, 51571056, 51771048, 51601033), the 863 Program of China (Grant No. 2015AA034101) and the Fundamental Research Funds for the Central Universities of

China (Grants No. N180204013) are acknowledged. Naifu Zou is grateful to the China Scholarship Council for the PhD grant during his study in France. 


\section{Appendices}

\begin{tabular}{|c|c|c|c|}
\hline Twinning & $\begin{array}{c}\text { A-C or B-D } \\
\text { (Type-I) }\end{array}$ & $\begin{array}{c}\text { A-B or C-D } \\
\text { (Type-II) }\end{array}$ & $\begin{array}{l}\text { A-D or B-C } \\
\text { (Compound) }\end{array}$ \\
\hline$K_{1}$ & $(12 \overline{10})_{7 \mathrm{M}}$ & $\left(1.06212 \overline{9.3785}_{7 \mathrm{M}}\right.$ & $\left(\begin{array}{lll}1 & 0 & 10\end{array}\right)_{7 \mathrm{M}}$ \\
\hline$K_{2}$ & $\left(\begin{array}{ll}(1.0621 & 29.3785)_{7 \mathrm{M}}\end{array}\right.$ & $\left(\begin{array}{lll}\overline{1} & 2 & 10\end{array}\right)_{7 \mathrm{M}}$ & $\left(\begin{array}{lll}\overline{1} & 0 & 10\end{array}\right)_{7 \mathrm{M}}$ \\
\hline$\eta_{1}$ & {$\left[\begin{array}{llll}\overline{10.5541} & 10 & 0.9446\end{array}\right]_{7 \mathrm{M}}$} & {$\left[\begin{array}{llll}\overline{10} & 10 & 1\end{array}\right]_{7 \mathrm{M}}$} & {$\left[\begin{array}{lll}\overline{10} & 0 & 1\end{array}\right]_{7 \mathrm{M}}$} \\
\hline$\eta_{2}$ & {$\left[\begin{array}{lll}10 & 10 & \overline{1}\end{array}\right]_{7 \mathrm{M}}$} & {$\left[\begin{array}{lll}10.5541 & 10 & \overline{0.9446}\end{array}\right]_{7 \mathrm{M}}$} & {$\left[\begin{array}{lll}1 & 0 & 1\end{array}\right]_{7 \mathrm{M}}$} \\
\hline$P$ & $(\overline{1} \overline{0.0570} \overline{10.5699})_{7 \mathrm{M}}$ & $\left(\begin{array}{llll}\overline{1} & 0.0570 & \overline{10.5699}\end{array}\right)_{7 \mathrm{M}}$ & $\left(\begin{array}{lll}0 & 1 & 0\end{array}\right)_{7 \mathrm{M}}$ \\
\hline$s$ & 0.2299 & 0.2299 & 0.0135 \\
\hline
\end{tabular}

Table A.1 Twinning elements $K_{1}, K_{2}, \eta_{1}, \eta_{2}, P$ and $s$ of Type-I, Type-II and compound TrF-twins.

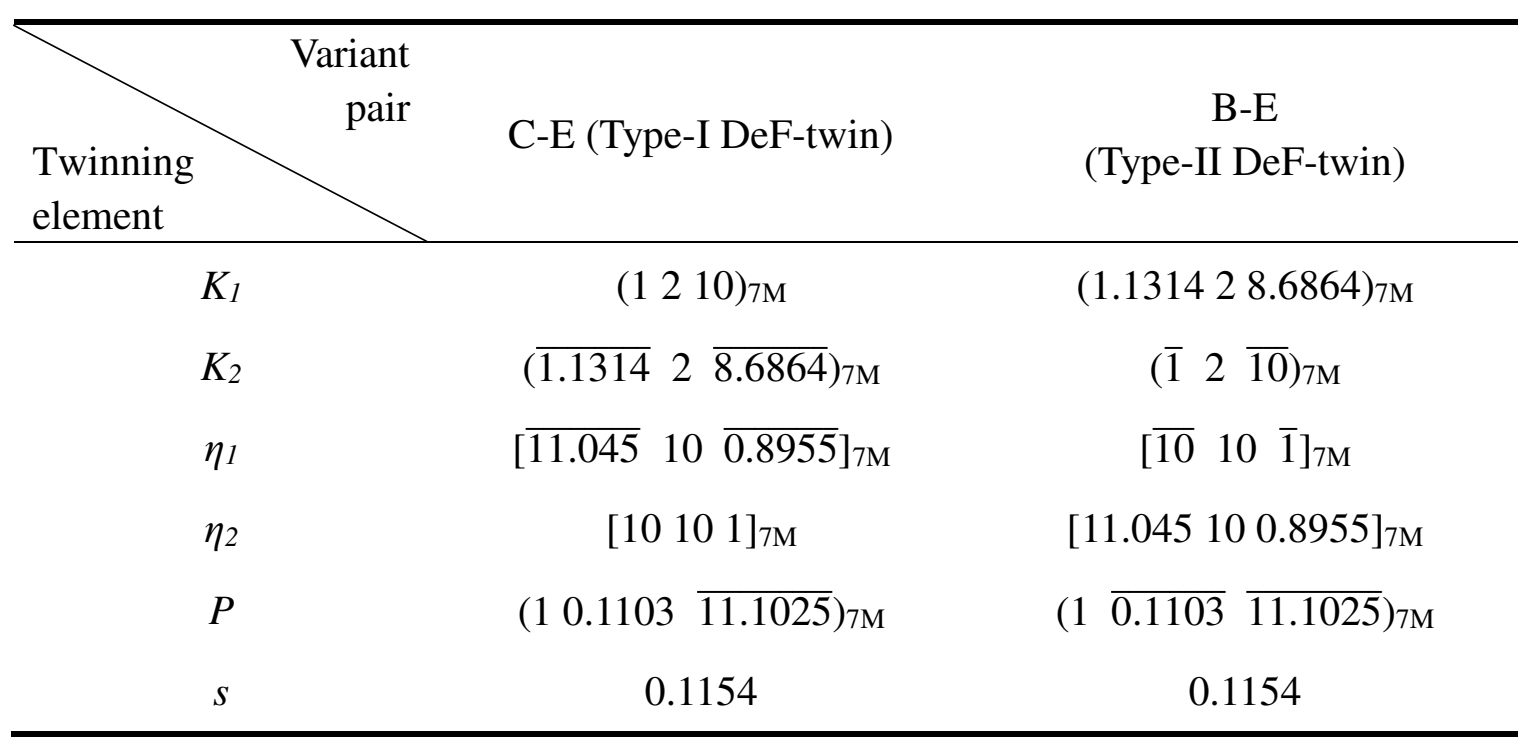

Table A.2 Twinning elements $K_{1}, K_{2}, \eta_{1}, \eta_{2}, P$ and $s$ of Type-I and Type-II DeF-twins. 


\section{References}

[1] A. Sozinov, N. Lanska, A. Soroka, W. Zou, 12\% magnetic field-induced strain in Ni-Mn-Ga-based non-modulated martensite, Appl. Phys. Lett. 102(2) (2013) 021902.

[2] A. Sozinov, A. A. Likhachev, N. Lanska, K. Ullakko, Giant magnetic-field-induced strain in Ni-Mn-Ga seven-layered martensitic phase, Appl. Phys. Lett. 80(10) (2002) 1746-1748.

[3] Z. B. Li, Y. W. Jiang, Z. Z. Li, C. F. Sanchez Valdes, J. L. Sanchez Llamazares, B. Yang, Y. D. Zhang, C. Esling, X. Zhao, L. Zuo, Phase transition and magnetocaloric properties of $\mathrm{Mn}_{50} \mathrm{Ni}_{42-\mathrm{x}} \mathrm{Co}_{\mathrm{x}} \mathrm{Sn}_{8}(0 \leqslant \mathrm{x} \leqslant 10)$ melt-spun ribbons, IUCrJ 5 (2018) 54-66.

[4] H. E. Karaca, I. Karaman, B. Basaran, Y. Ren, Y. I. Chumlyakov, H. J. Maier, Magnetic Field-Induced Phase Transformation in Ni-Mn-Co-In Magnetic Shape-Memory Alloys-A New Actuation Mechanism with Large Work Output, Adv. Funct. Mater. 19(7) (2009) 983-998.

[5] J. A. Monroe, I. Karaman, B. Basaran, W. Ito, R. Y. Umetsu, R. Kainuma, K. Koyama, Y. I. Chumlyakov, Direct measurement of large reversible magnetic-field-induced strain in Ni-Co-Mn-In metamagnetic shape memory alloys, Acta Mater. 60(20) (2012) 6883-6891.

[6] L. Straka, O. Heczko, H. Seiner, N. Lanska, J. Drahokoupil, A. Soroka, S. Fähler, H. Hänninen, A. Sozinov, Highly mobile twinned interface in 10M modulated Ni-Mn-Ga martensite: Analysis beyond the tetragonal approximation of lattice, Acta Mater. 59(20) (2011) 7450-7463.

[7] L. Straka, A. Soroka, H. Seiner, H. Hänninen, A. Sozinov, Temperature dependence of twinning stress of Type-I and Type-II twins in 10M modulated Ni-Mn-Ga martensite, Scr. 
Mater. 67(1) (2012) 25-28.

[8] N. Zárubová, Y. Ge, O. Heczko, S. P. Hannula, In-situ TEM study of deformation twinning in Ni-Mn-Ga non-modulated martensite, Acta Mater. 61(14) (2013) 5290-5299.

[9] C. Witherspoon, P. Zheng, M. Chmielus, S. C. Vogel, D. C. Dunand, P. Müllner, Texture and training of magnetic shape memory foam, Acta Mater. 61(6) (2013) 2113-2120.

[10]I. Aaltio, Y. Ge, S. P. Hannula, Twinning in shear and uniaxial loading in five layered martensite Ni-Mn-Ga single crystals, Eur. Phys. J. B 86(2) (2013) 49.

[11]A. Saren, D. Musiienko, A. R. Smith, K. Ullakko, Pulsed magnetic field-induced single twin boundary motion in Ni-Mn-Ga 5M martensite: a laser vibrometry characterization, Scr. Mater. 113 (2016) 154-157.

[12]I. Aaltio, O. Söderberg, Y. Ge, S. P. Hannula, Twin boundary nucleation and motion in Ni-Mn-Ga magnetic shape memory material with a low twinning stress, Scr. Mater. 62(1) (2010) 9-12.

[13]E. Faran, I. Benichou, S. Givli, D. Shilo, The effects of magnetic and mechanical microstructures on the twinning stress in Ni-Mn-Ga, J. Appl. Phys. 118(24) (2015) 244104.

[14]R. Chulist, W. Skrotzki, C. G. Oertel, A. Böhm, M. Pötschke, Change in microstructure during training of a $\mathrm{Ni}_{50} \mathrm{Mn}_{29} \mathrm{Ga}_{21}$ bicrystal, Scr. Mater. 63(5) (2010) 548-551.

[15]M. J. Szczerba, R. Chulist, Detwinning of a non-modulated Ni-Mn-Ga martensite: from self-accommodated microstructure to single crystal, Acta Mater. 85 (2015) 67-73.

[16]B. Muntifering, L. Kovarik, N. D. Browning, R. C. Pond, W. B. Knowlton, P. Müllner, Stress-assisted removal of conjugation boundaries in non-modulated Ni-Mn-Ga by 
coordinated secondary twinning, J. Mater. Sci. 51(1) (2016) 457-466.

[17]Z. H. Nie, R. L. Peng, S. Johansson, E. C. Oliver, Y. Ren, Y. D. Wang, Y. D. Liu, J. N. Deng, L. Zuo, D. E. Brown, Direct evidence of detwinning in polycrystalline Ni-Mn-Ga ferromagnetic shape memory alloys during deformation, J. Appl. Phys. 104(10) (2008) 103519.

[18]P. Müllner, V. A. Chernenko, G. Kostorz, Large cyclic magnetic-field-induced deformation in orthorhombic (14M) Ni-Mn-Ga martensite, J. Appl. Phys. 95(3) (2004) $1531-1536$.

[19] Y. C. Dai, L. Hou, Y. Fautrelle, Z. B. Li, C. Esling, Z. M. Ren, X. Li, Detwinning process of martensite in $\mathrm{Ni}_{58} \mathrm{Mn}_{25} \mathrm{Ga}_{17}$ as a high temperature shape memory alloy under uniaxial compression, Inter. J. Plast. 103 (2018) 203-213.

[20]A. K. Kanjarla, P. Van Houtte, L. Delannay, Assessment of plastic heterogeneity in grain interaction models using crystal plasticity finite element method, Inter. J. Plast. 26(8) (2010) 1220-1233.

[21]P. Thamburaja, H. Pan, F. S. Chau, The evolution of microstructure during twinning: Constitutive equations, finite-element simulations and experimental verification, Inter. J. Plast. 25 (2009) 2141-2168.

[22]Q. Xie, A. Van Bael, J. Sidor, J. Moerman, P. Van Houtte, A new cluster-type model for the simulation of textures of polycrystalline metals, Acta Mater. 69 (2014) 175-186.

[23]J. Wang, H. Sehitoglu, H. J. Maier, Dislocation slip stress prediction in shape memory alloys, Inter. J. Plast. 54 (2014) 247-266.

[24]P. Müllner, A. H. King, Deformation of hierarchically twinned martensite, Acta Mater. 
58(16) (2010) 5242-5261.

[25]K. Bhattacharya, Microstructure of martensite: why it forms and how it gives rise to the shape-memory effect (Vol. 2), Oxford University Press, 2003.

[26]N. F. Zou, Z. B. Li, Y. D. Zhang, B. Yang, X. Zhao, C. Esling, L. Zuo, Plastic deformation of Ni-Mn-Ga 7M modulated martensite by twinning \& detwinning and intermartensitic transformation, Inter. J. Plast. 100 (2018) 1-13.

[27]E. Pagounis, R. Chulist, T. Lippmann, M. Laufenberg, W. Skrotzki, Structural modification and twinning stress reduction in a high-temperature Ni-Mn-Ga magnetic shape memory alloy, Appl. Phys. Lett. 103(11) (2013) 111911.

[28] Y. Ge, N. Zárubová, O. Heczko, S. P. Hannula, Stress-induced transition from modulated 14M to non-modulated martensite in Ni-Mn-Ga alloy, Acta Mater. 90 (2015) 151-160.

[29]U. Gaitzsch, M. Pötschke, S. Roth, N. Mattern, B. Rellinghaus, L. Schultz, Structure formation in martensitic $\mathrm{Ni}_{50} \mathrm{Mn}_{30} \mathrm{Ga}_{20}$ MSM alloy, J. Alloy Compd. 443(1-2) (2007) 99104.

[30]L. Huang, D. Y. Cong, Z. L. Wang, Z. H. Nie, Y. H. Dong, Y. Zhang, Y. Ren, Y. D. Wang, Direct evidence for stress-induced transformation between coexisting multiple martensites in a Ni-Mn-Ga multifunctional alloy, J. Phys. D: Appl. Phys. 48(26) (2015) 265304.

[31]U. Gaitzsch, M. Pötschke, S. Roth, B. Rellinghaus, L. Schultz, Mechanical training of polycrystalline 7M Ni50 $\mathrm{Mn}_{30} \mathrm{Ga}_{20}$ magnetic shape memory alloy, Scr. Mater. 57(6) (2007) $493-495$.

[32]L. Righi, F. Albertini, E. Villa, A. Paoluzi, G. Calestani, V. Chernenko, S. Besseghini, C. 
Ritter, F. Passaretti, Crystal structure of 7M modulated Ni-Mn-Ga martensitic phase, Acta Mater. 56(16) (2008) 4529-4535.

[33]Z. B. Li, Y. D. Zhang, C. Esling, X. Zhao, L. Zuo, Determination of the orientation relationship between austenite and incommensurate $7 \mathrm{M}$ modulated martensite in Ni-Mn-Ga alloys, Acta Mater. 59(7) (2011) 2762-2772.

[34]Z. B. Li, Y. D. Zhang, C. Esling, X. Zhao, Y. D. Wang, L. Zuo, New approach to twin interfaces of modulated martensite, J. Appl. Cryst. 43(3) (2010) 617-622.

[35]Z. B. Li, B. Yang, Y. D. Zhang, C. Esling, N. F. Zou, X. Zhao, L. Zuo, Crystallographic insights into the intermartensitic transformation in Ni-Mn-Ga alloys, Acta Mater. 74 (2014) 9-17.

[36]M. Hoelzel, W. M. Gan, M. Hofmann, C. Randau, G. Seidl, P. Jüttner, W. W. Schmahl, Rotatable multifunctional load frames for neutron diffractometers at FRM II-design, specifications and applications, Nuclear Instruments and Methods in Physics Research Section A: Accelerators, Spectrometers, Detectors and Associated Equipment 711 (2013) 101-105.

[37]C. Randau, U. Garbe, H. G. Brokmeier, StressTextureCalculator: a software tool to extract texture, strain and microstructure information from area-detector measurements, J. Appl. Cryst. 44(3) (2011) 641-646.

[38]Z. B. Li, Y. D. Zhang, C. Esling, H. Yang, J. J. Wang, C. S. He, X. Zhao, L. Zuo, Application of EBSD to the Crystallographic Investigation on Ni-Mn-Ga Alloys, Mater. Sci. Forum 706 (2012) 1879-1884.

[39]M. Chmielus, K. Rolfs, R. Wimpory, W. Reimers, P. Müllner, R. Schneider, Effects of 
surface roughness and training on the twinning stress of Ni-Mn-Ga single crystals, Acta Mater. 58(11) (2010) 3952-3962.

[40]Z. B. Li, Y. D. Zhang, C. Esling, X. Zhao, L. Zuo, Evidence for a monoclinic incommensurate superstructure in modulated martensite, Acta Mater. 60(20) (2012) 6982-6990.

[41]H. L. Yan, B. Yang, Y. D. Zhang, Z. B. Li, C. Esling, X. Zhao, L. Zuo, Variant organization and mechanical detwinning of modulated martensite in Ni-Mn-In metamagnetic shape-memory alloys, Acta Mater. 111 (2016) 75-84.

[42]Y. D. Zhang, Z. B. Li, C. Esling, J. Muller, X. Zhao, L. Zuo, A general method to determine twinning elements, J. Appl. Cryst. 43(6) (2010) 1426-1430.

[43] Y. D. Zhang, C. Esling, X. Zhao, L. Zuo, Indirect two-trace method to determine a faceted low-energy interface between two crystallographically correlated crystals, J. Appl. Cryst. 40(3) (2007) 436-440. 


\section{Figure captions}

Fig. 1 (a) Schematic representation of the sample position with respect to the compressive load direction (LD), the incident beam (BD) and the diffracted beam detected by the 2D detector. In the figure, the geometrical centers of the sample and the 2D detector and the axial axis of the incident beam constitute the equatorial plane (outlined with the black circle). The loading axis tilts within the plane (outlined with the red circle) perpendicular to the equatorial plane and bisecting the angle between the incident beam and the detected diffracted beam. The angle between LD and the equatorial plane is $\chi$ and the rotation angle around LD is $\omega$. (b) Home-made compression device adapted to the SEM sample stage. The load was applied by screwing forward the movable crosshead, and the displacement of the crosshead was measured by a digital caliper.

Fig. $2\left\{\begin{array}{lll}\overline{1} & 2 & 10\end{array}\right\}_{7 \mathrm{M}}(\mathrm{a}),\left\{\begin{array}{lll}1 & 0 & 10\end{array}\right\}_{7 \mathrm{M}}(\mathrm{b})$ and $\left\{\begin{array}{lll}1 & 2 & 10\end{array}\right\}_{7 \mathrm{M}}$ (c) pole figures of the as-annealed polycrystalline Ni-Mn-Ga. The reference frame in polar coordinate is displayed in (d). BD stands for incident beam direction and SD the solidification direction.

Fig. 3 SEM backscattered electron (BSE) micrograph (a) and EBSD micrograph of the as-annealed Ni50Mn30Ga20 alloy fabricated by directional solidification. Original austenite grain boundaries are marked with the red lines, and martensite colonies are outlined with black lines. In (b), martensite variants are colored according to their Euler angle values. The same color represents the same orientation. One example variant colony enclosed in the yellow rectangle frame in (b) is further magnified in (c) with the corresponding $\left\{\begin{array}{lll}\overline{1} & 2 & 10\end{array}\right\}_{7 \mathrm{M}}$, 
$\left\{\begin{array}{lll}1 & 0 & 10\end{array}\right\}_{7 \mathrm{M}}$ and $\left\{\begin{array}{lll}1 & 2 & 10\end{array}\right\}_{7 \mathrm{M}}$ plane pole figures of the variants. The four variants in the colony are indicated with A, B, C and D. The traces of the three planes are indicated with the yellow lines.

Fig. 4 Stress-strain curves during unidirectional compression at room temperature (a), and the corresponding neutron diffraction patterns $\left(\left(b_{1}\right)\right.$ for $S 1,\left(b_{2}\right)$ for $S 2$ and $\left(b_{3}\right)$ for $\left.S 3\right)$ measured within $2 \theta$ of $35-49^{\circ}$ or $55-69^{\circ}$ at various deformation stages indicated with the consistent colors on the stress-strain curve. The evolution of $\left\{\begin{array}{llll}2 & 0 & 0\end{array}\right\}_{\mathrm{NM}}$ is highlighted with the rectangular frame in $\left(b_{3}\right)$ and further magnified in the insert.

Fig. 5 SEM/EBSD orientation micrographs of the sample area acquired at reductions of $0 \%$ $\left(\mathrm{a}_{1}\right), 2 \%\left(\mathrm{~b}_{1}\right), 3 \%\left(\mathrm{c}_{1}\right), 4.5 \%\left(\mathrm{~d}_{1}\right)$ and $5.5 \%\left(\mathrm{e}_{1}\right)$. The corresponding area fractions of variants with corresponding standard deviations as area fraction spread are displayed in $\left(\mathrm{a}_{2}\right)-\left(\mathrm{e}_{2}\right)$. The compressive load was applied parallel to $\mathrm{X}$ axis that is also parallel to the solidification direction (SD).

Fig. 6 Atomic correspondences of $7 \mathrm{M}$ variant $\mathrm{O}$ and $\mathrm{E}$ and $\mathrm{NM}$ martensite under the intermartensitic transformation ORs. $\boldsymbol{n}$ represents the OR plane normal.

Fig. 7 (a) Pole figure of the twining plane of twin variant pair O-E (compound DeF-twin, $\left\{\begin{array}{l}0 \\ 0\end{array}\right.$ $10\}_{7 \mathrm{M}}$ ) and (b) the EBSD IPF micrograph of the microstructure of the variant pair O-E. In the 
figures the O-E interface traces are marked with the black straight lines. The dashed line connecting the origin of the pole figure and the pole of the interface in (a) is perpendicular to the trace of interface.

Fig. 8 EBSD $\left\{\begin{array}{lll}0 & 2 & 0\end{array}\right\}_{7 \mathrm{M}}$ pole figures of (a) the initial state and (b) the deformed state $(5.5 \%$ in reduction). The $\mathrm{X}$ direction is parallel to the compression direction (LD).

Fig. $9\left\{\begin{array}{lll}0 & 2 & 0\end{array}\right\}_{7 M}$ pole figures from through-volume neutron diffraction measurements of the initial un-deformed state (a) and the deformed states corresponding to $2 \%$ (b), $4 \%$ (c) and $6 \%$ (d) in reduction. BD is the beam direction and $\mathrm{LD}$ is the compression direction. 

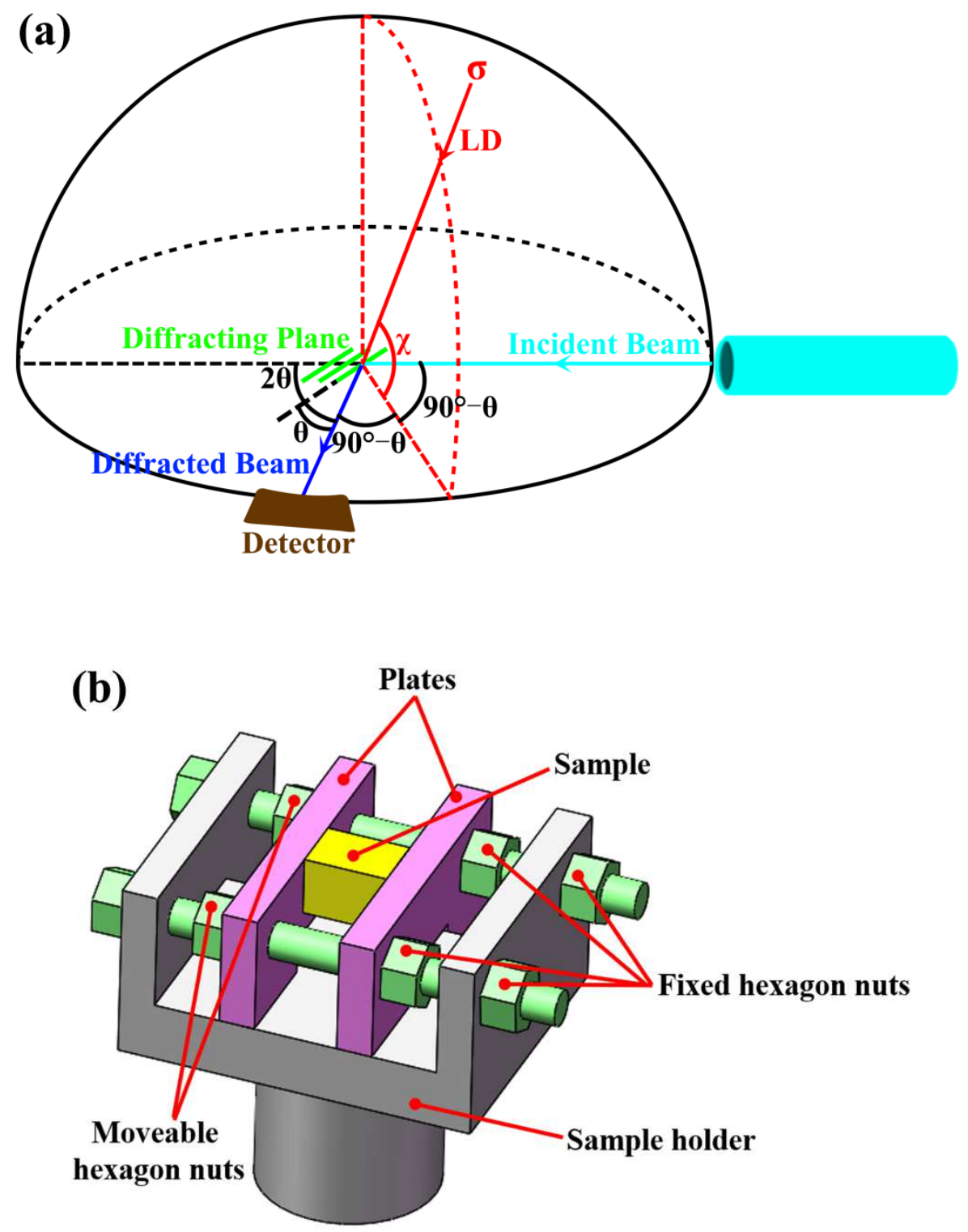

Fig. 1 (a) Schematic representation of the sample position with respect to the compressive load direction (LD), the incident beam (BD) and the diffracted beam detected by the $2 \mathrm{D}$ detector. In the figure, the geometrical centers of the sample and the 2D detector and the axial axis of the incident beam constitute the equatorial plane (outlined with the black circle). The loading axis tilts within the plane (outlined with the red circle) perpendicular to the equatorial plane and bisecting the angle between the incident beam and the detected diffracted beam. The angle between LD and the equatorial plane is $\chi$ and the rotation angle around LD is $\omega$. (b) Homemade compression device adapted to the SEM sample stage. The load was applied by screwing forward the movable crosshead, and the displacement of the crosshead was measured by a digital caliper. 

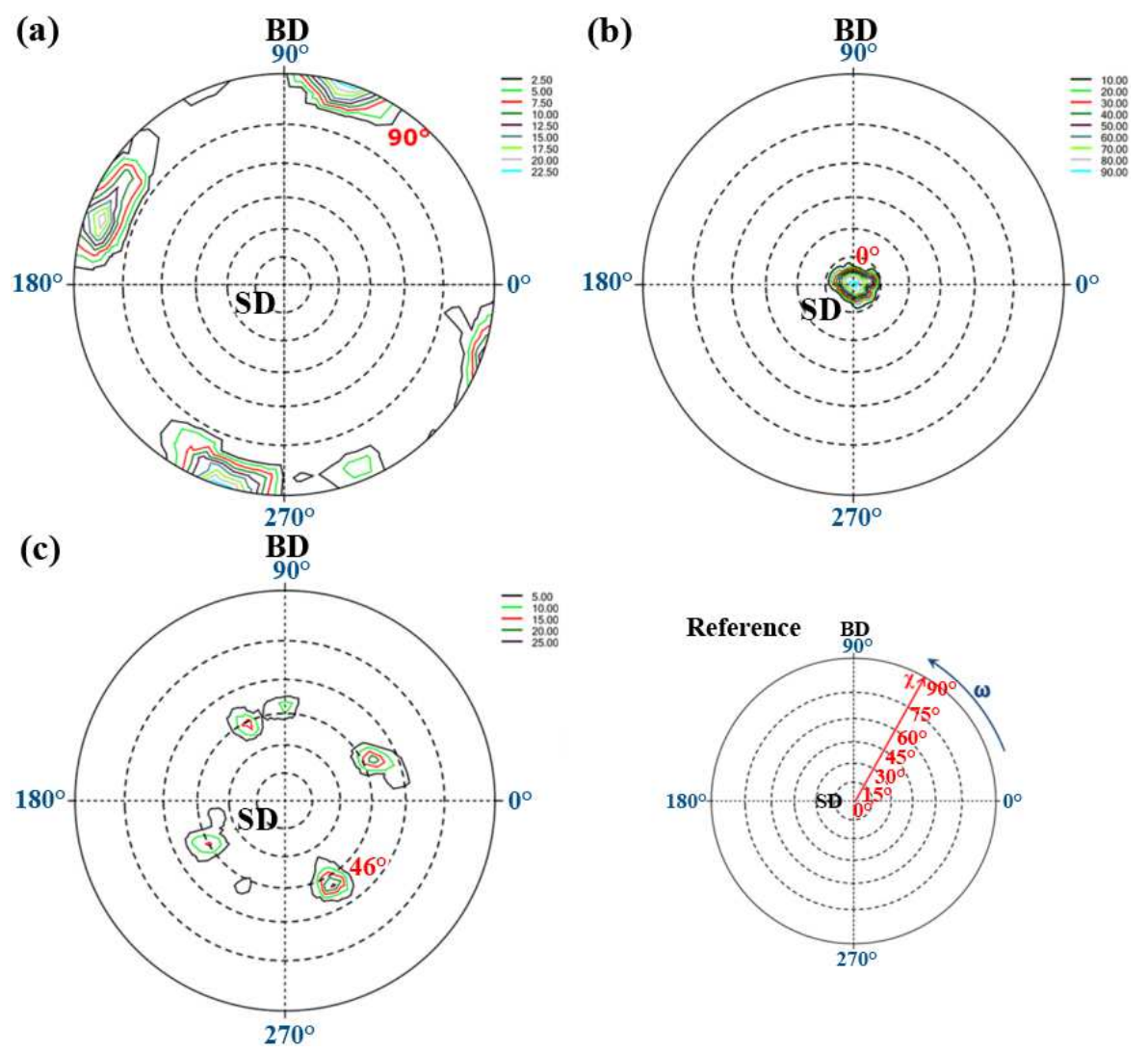

Fig. $2\left\{\begin{array}{llll}1 & 2 & 10\end{array}\right\}_{7 \mathrm{M}}(\mathrm{a}),\left\{\begin{array}{lll}1 & 0 & 10\end{array}\right\}_{7 \mathrm{M}}(\mathrm{b})$ and $\left\{\begin{array}{lll}1 & 2 & 10\end{array}\right\}_{7 \mathrm{M}}$ (c) pole figures of the as-annealed polycrystalline Ni-Mn-Ga. The reference frame in polar coordinate is displayed in (d). BD stands for incident beam direction and SD the solidification direction. 

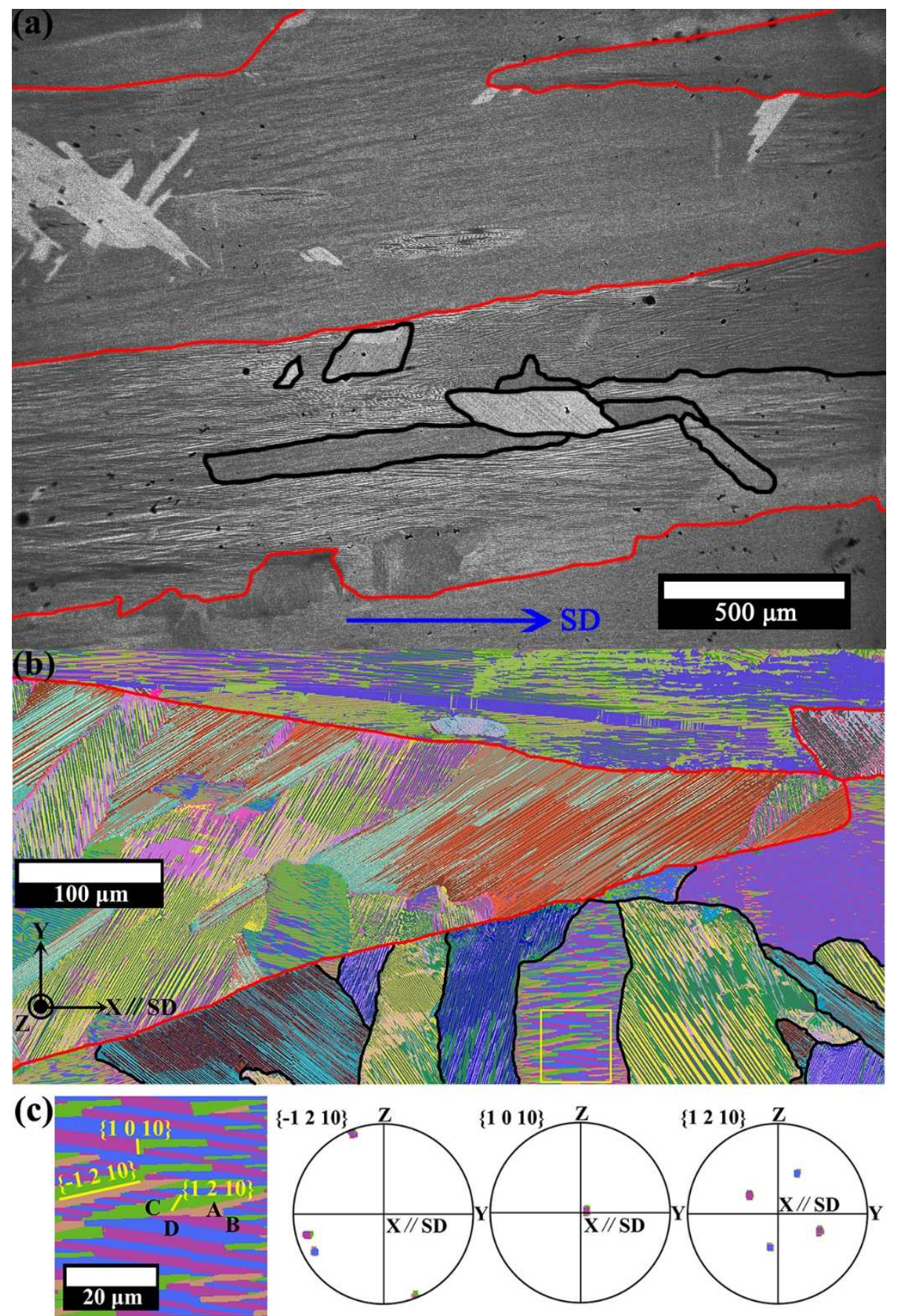

Fig. 3 SEM backscattered electron (BSE) micrograph (a) and EBSD micrograph of the as-annealed Ni50Mn30Ga20 alloy fabricated by directional solidification. Original austenite grain boundaries are marked with the red lines, and martensite colonies are outlined with black lines. In (b), martensite variants are colored according to their Euler angle values. The same color represents the same orientation. One example variant colony enclosed in the yellow rectangle frame in (b) is further magnified in (c) with the 
corresponding $\left\{\begin{array}{lll}\overline{1} & 2 & 10\end{array}\right\}_{7 \mathrm{M}},\left\{\begin{array}{lll}1 & 0 & 10\end{array}\right\}_{7 \mathrm{M}}$ and $\left\{\begin{array}{lll}1 & 2 & 10\end{array}\right\}_{7 \mathrm{M}}$ plane pole figures of the variants. The four variants in the colony are indicated with A, B, C and D. The traces of the three planes are indicated with the yellow lines. 


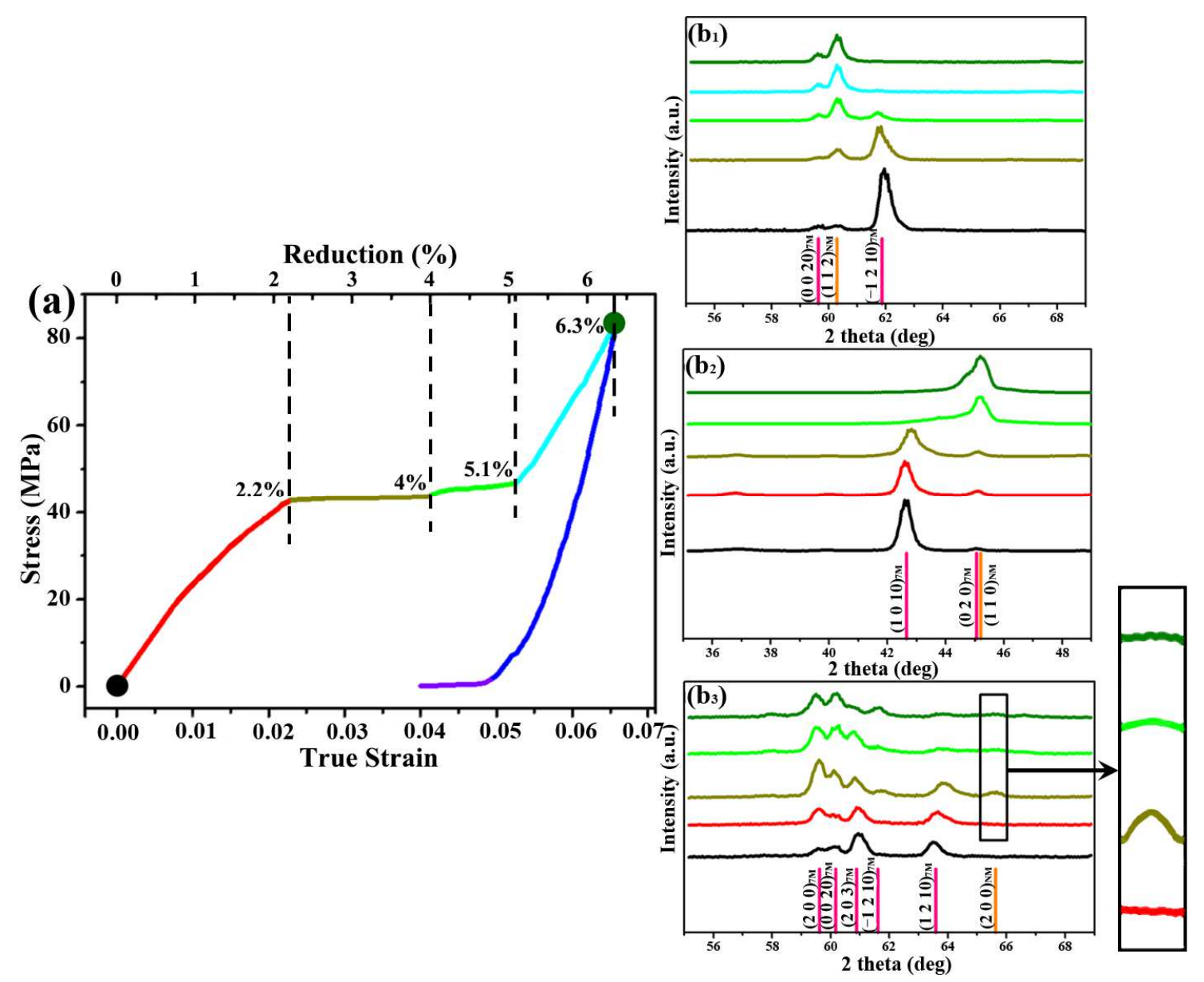

Fig. 4 Stress-strain curves during unidirectional compression at room temperature (a), and the corresponding neutron diffraction patterns $\left(\left(b_{1}\right)\right.$ for $S 1,\left(b_{2}\right)$ for $S 2$ and $\left(b_{3}\right)$ for $\left.S 3\right)$ measured within $2 \theta$ of $35-49^{\circ}$ or $55-69^{\circ}$ at various deformation stages indicated with the consistent colors on the stress-strain curve. The evolution of $\{200\}_{\mathrm{NM}}$ is highlighted with the rectangular frame in $\left(b_{3}\right)$ and further magnified in the insert. 


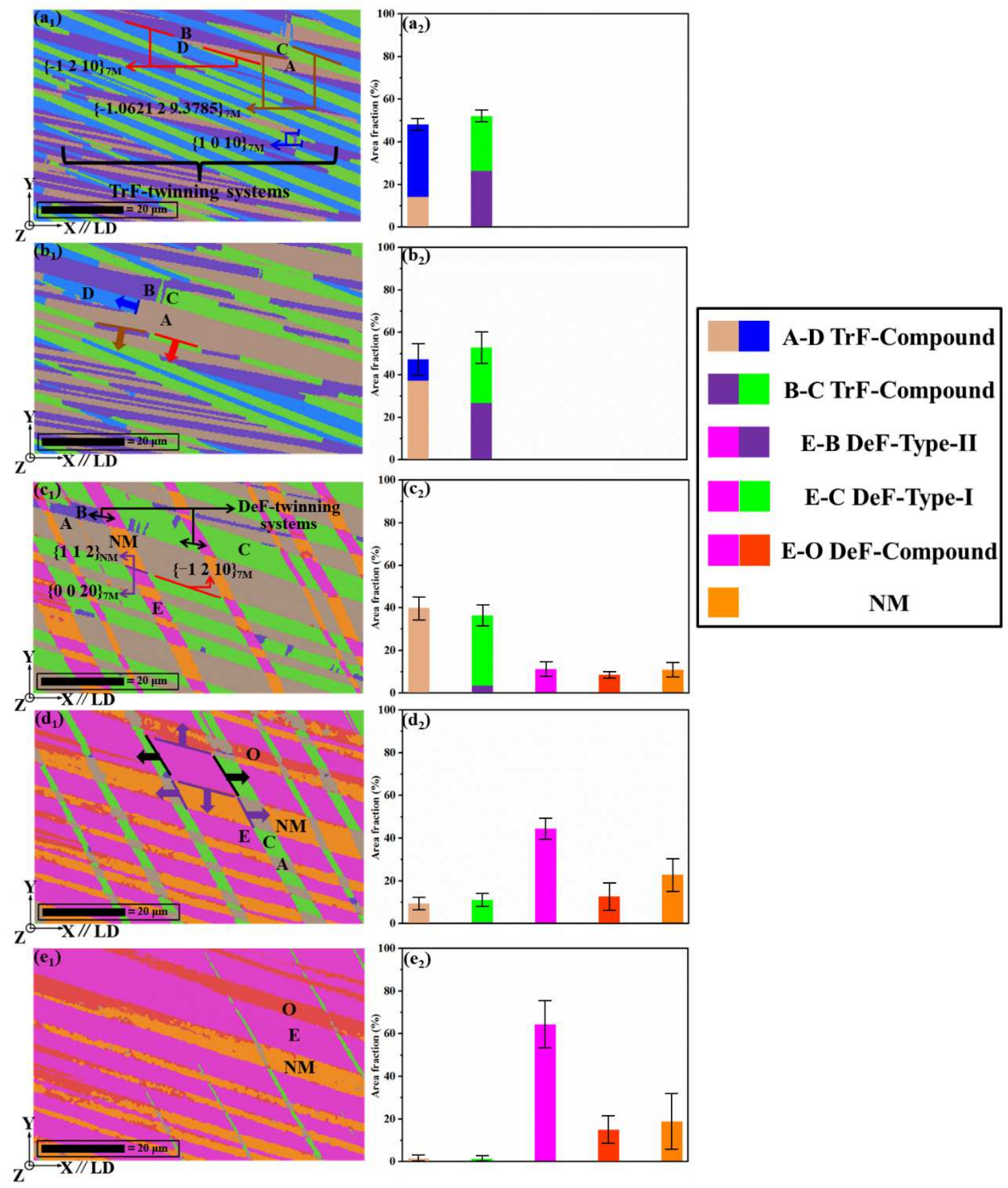

Fig. 5 SEM/EBSD orientation micrographs of the sample area acquired at reductions of $0 \%$ $\left(a_{1}\right), 2 \%\left(b_{1}\right), 3 \%\left(c_{1}\right), 4.5 \%\left(d_{1}\right)$ and $5.5 \%\left(e_{1}\right)$. The corresponding area fractions of variants with corresponding standard deviations as area fraction spread are displayed in $\left(a_{2}\right)-\left(e_{2}\right)$. The compressive load was applied parallel to $\mathrm{X}$ axis that is also parallel to the solidification direction (SD). 


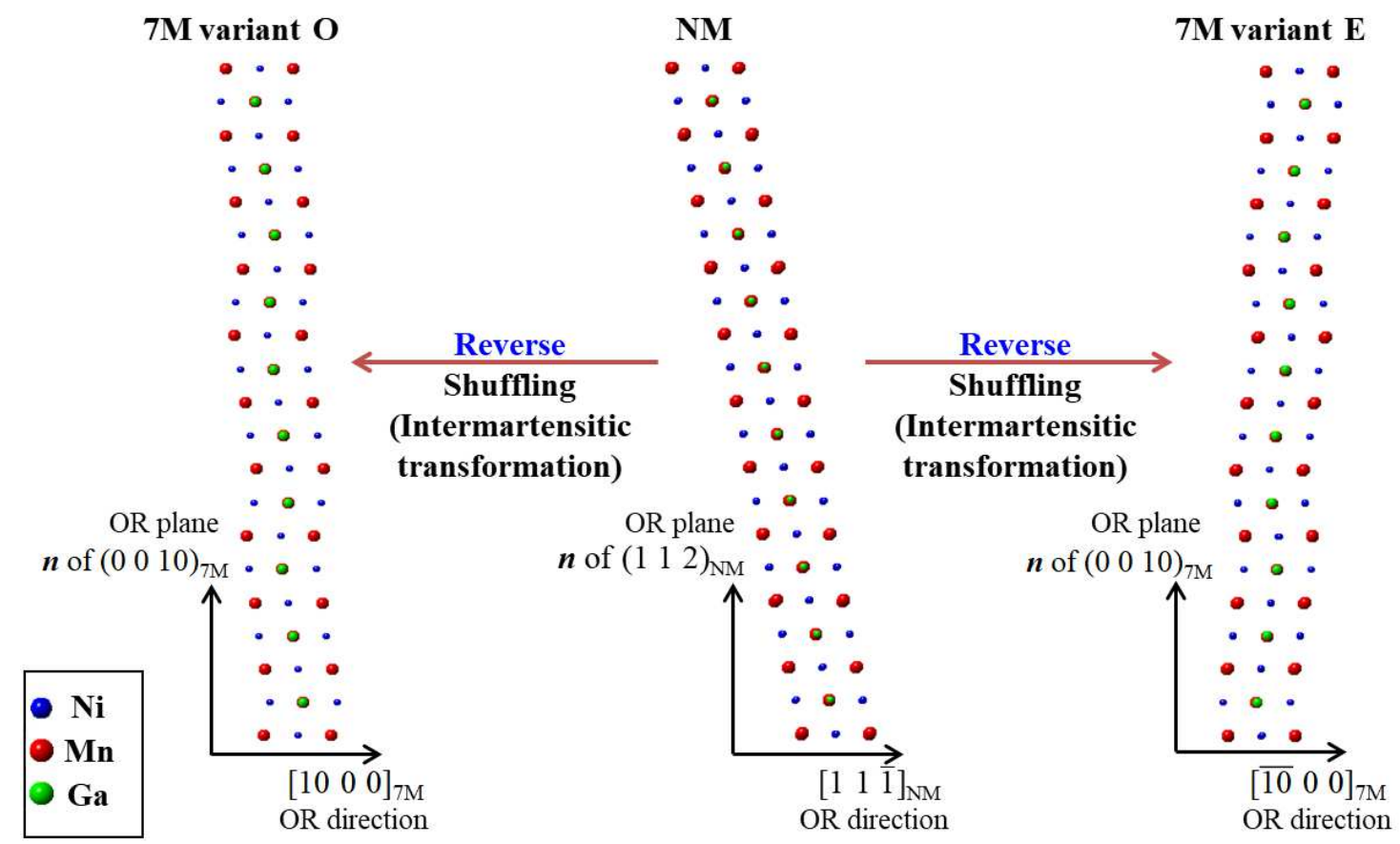

Fig. 6 Atomic correspondences of $7 \mathrm{M}$ variant $\mathrm{O}$ and $\mathrm{E}$ and $\mathrm{NM}$ martensite under the intermartensitic transformation ORs. $\boldsymbol{n}$ represents the OR plane normal. 


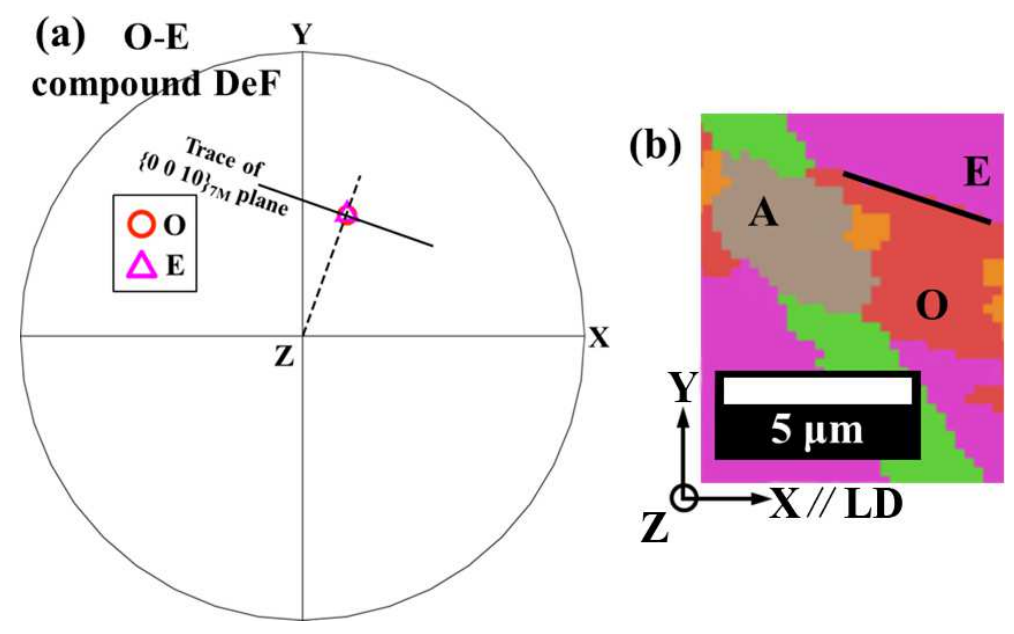

Fig. 7 (a) Pole figure of the twining plane of twin variant pair O-E (compound DeF-twin, $\begin{cases}0 & 0\end{cases}$ $10\}_{7 \mathrm{M}}$ ) and (b) the EBSD IPF micrograph of the microstructure of the variant pair O-E. In the figures the O-E interface traces are marked with the black straight lines. The dashed line connecting the origin of the pole figure and the pole of the interface in (a) is perpendicular to the trace of interface. 


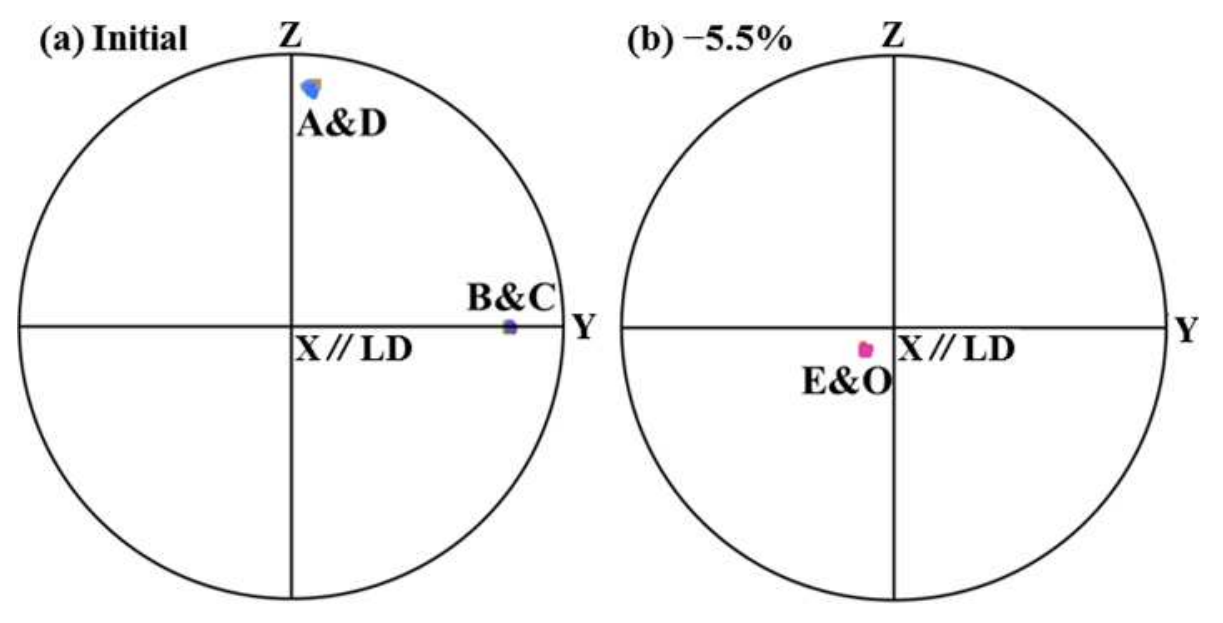

Fig. 8 EBSD $\left\{\begin{array}{lll}0 & 2 & 0\end{array}\right\}_{7 \mathrm{M}}$ pole figures of (a) the initial state and (b) the deformed state $(5.5 \%$ in reduction). The $\mathrm{X}$ direction is parallel to the compression direction (LD). 

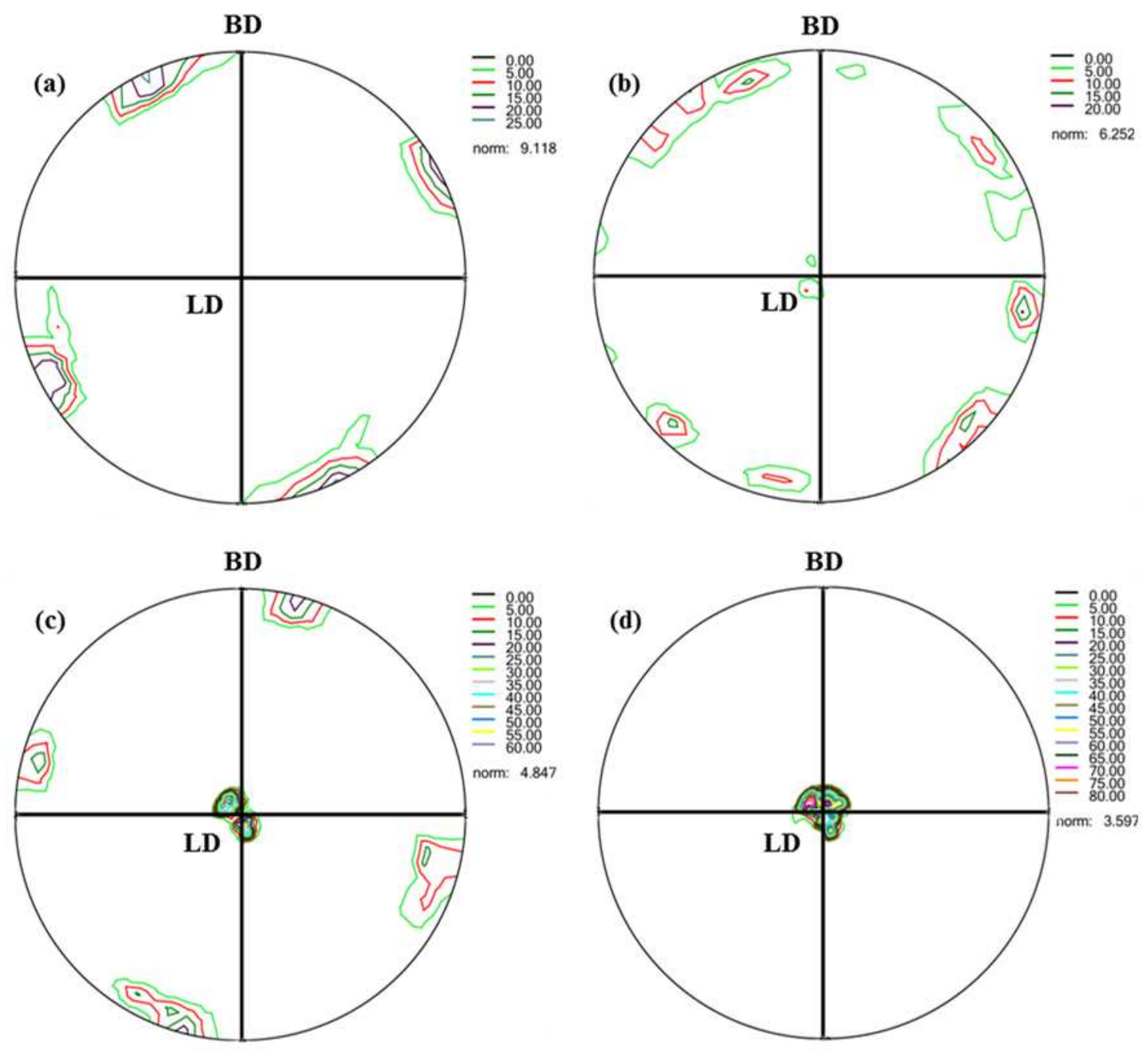

Fig. $9\left\{\begin{array}{lll}0 & 2 & 0\end{array}\right\}_{7 \mathrm{M}}$ pole figures from through-volume neutron diffraction measurements of the initial un-deformed state (a) and the deformed states corresponding to $2 \%$ (b), $4 \%$ (c) and $6 \%$ (d) in reduction. BD is the beam direction and LD is the compression direction. 


\section{Neutron diffraction patterns}

Stress-strain curve $\&$ microstructure

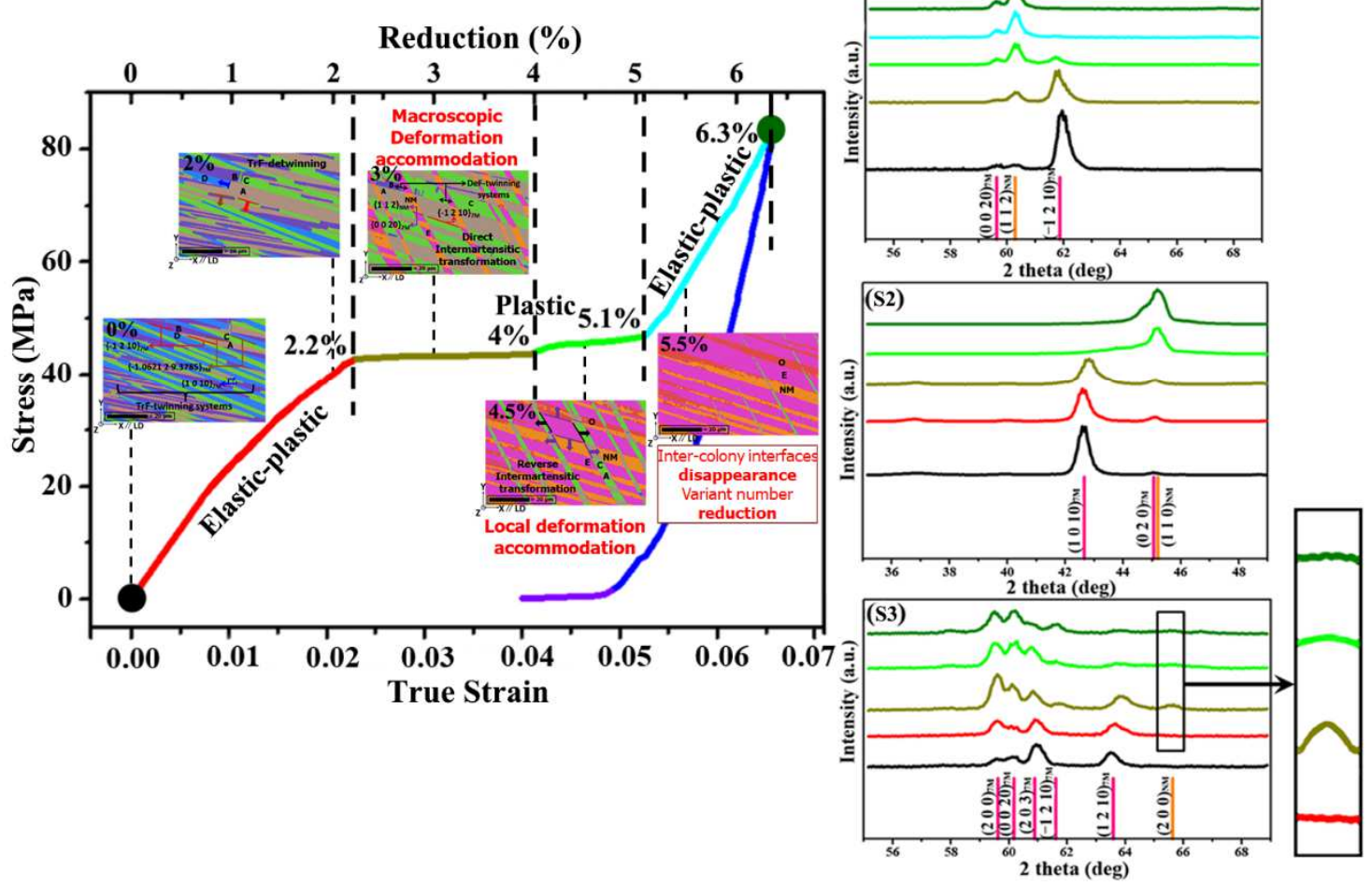

